\title{
A CHECKLIST OF TASMANIAN LICHENS
}

\author{
by Gintaras Kantvilas
}

KANTVILAS, G., 1989 (31:x): A checklist of Tasmanian lichens. Pap. Proc. R. Soc. Tasm. 123:-67 -85. https://doi.org/10.26749/rstpp.123.67 ISSN 0080-4703. Tasmanian Herbarium, GPO Box 252C, Hobart, Tasmania, Australia 7001.

This list records 633 lichen species plus 22 infraspecific taxa in 173 genera from Tasmania and the Bass Strait islands. Fourteen species are new records for Tasmania: Arthroraphis citrinella, Cetraria australiensis, Coccotrema porinopsis, Hyperphyscia adglutinata, Lecanora blanda, Neofuscelia verrucella, Pertusaria superba, Phaeographis australiensis, Relicina subnigra, Rinodina bischoffii, Siphulastrum mamillatum, Sphaerophorus imshaugii, Stereocaulon gregarium and Usnea contexta. A list of deleted names based on unreliable sources, misidentifications or misinterpretations of early literature is also provided.

Key Words: lichens, Tasmania.

\section{INTRODUCTION}

The last checklist dealing exclusively with the lichen flora of Tasmania was published by Wetmore (1963). That work cited all the hitherto published records of Tasmanian species (primarily from 19thcentury literature) and listed 421 species in 82 genera. The last two decades have seen a major resurgence in lichenology, and many significant advances in the taxonomy of the Tasmanian flora have hastened the need for a new checklist. For example, many genera and families in the Australian region have been recently revised, including the Caliciales (Tibell 1987), Cladia (Filson 1981), Cladonia sect. Cocciferae (Archer 1988a), Haematomma (Rogers 1982, 1985), Heterodea (Blackman et al. 1973, Filson 1978), Hypogymnia (Elix 1979), Knightiella (Galloway \& Elix 1980), Leioderma (Galloway \& Jorgensen 1987), Nephroma (White \& James 1988), Pannoparmelia (Bratt et al. 1976, Galloway 1978), Parmelia (Filson 1982, Galloway \& Elix 1983, 1984), Peltula (Filson 1988a), Pyxine (Rogers 1986), Ramalina (Stevens 1987), Rinodina (Mayrhofer 1984), Teloschistes and Xanthoria (Filson 1969), Umbilicaria (Blackman et al. 1974) and Xanthoparmelia (Elix et al. 1986). Similarly, unpublished work by A.W. Archer and by D. Verdon has helped to clarify the genera Cladonia and Leptogium respectively.

Corresponding studies on the New Zealand lichen flora have also had a profound influence on Tasmanian lichenology in revising the taxonomy of many genera which are important in Tasmania. This work includes the pioneer studies of H.H. Allan, W. Martin and J. Murray (see Galloway 1974) as well as more recent work, e.g. Arthroraphis (Galloway \& Bartlett 1986), Baeomyces (Galloway 1980a),
Cladonia (Archer \& Bartlett 1986), Coccocarpia (Arvidsson \& Galloway 1979), the Graphidaceae (Hayward 1977), Lobaria (Galloway 1981), Pseudocyphellaria (Galloway 1983a, 1985a, 1988; Galloway \& James 1977, 1980; Galloway et al. 1983; Wilkins \& James 1979), Rinodina (Mayrhofer 1983), Stereocaulon (Galloway 1980b), Thysanothecium (Galloway \& Bartlett 1983), Xanthoparmelia (Galloway 1980c) and the recent Flora of New Zealand: Lichens (Galloway 1985b).

Collections from Tasmania have also figured in some recent world monographs, e.g. Cetraria (Kärnefelt 1979), Chrysothrix (Laundon 1981), Cladina (Ahti 1961, 1984), Coccocarpia (Arvidsson 1982), Collema (Degelius 1974), Degelia (Arvidsson \& Galloway 1981), the Megalosporaceae (Sipman 1983, 1986), Metus (Galloway \& James 1987), Parmelia (Esslinger 1977, Hale 1988), Pseudoparmelia (Hale 1976), Psoromidium (Galloway \& James 1985), Relicina (Hale 1975), Sphaerophorus (Ohlsson 1974, Tibell 1987) and Usnea subgen. Neuropogonon (Walker 1985), as well as in the studies on Alectoria (Hawksworth 1972), Cladonia (Ahti 1980) and Gymnoderma (Yoshimura 1973). This current work is based both on a review of existing 19th-century collections and the study of more recent collections.

New taxa from Tasmania have been published in recent years in the genera Acarospora and Catillaria (Räsänen 1944), Arthonia (Kantvilas \& Vezda 1988), Canoparmelia (Elix \& Johnston 1988), Cladia (Kantvilas \& Elix 1987), Cladonia (Archer 1982, 1984, 1985, 1986, 1987, 1988b), Diploschistes (Lumbsch 1987), Lichina (Henssen 1969), Lopadium (Döbbeler et al. 1985), Menegazzia (Kantvilas \& James 1987), Parmelina (Elix \&. Johnston 1986), Psoroma (James \& 
Henssen 1975), Pycnothelia (Galloway \& James 1987), Ramalina (Stevens 1986), Roccellina (Tehler 1983), Roccellinastrum (Henssen et al. 1982), Sarrameana (Vezda \& Kantvilas 1988), Siphula (Kantvilas 1987), Stereocaulon (Galloway et al. 1916), Xanthoparmelia (Elix \& Johnston 1987) andVezdaea (Döbbeler 1981), whilst the new Tasmanian genera Conotremopsis and Wawea were published by Vezda (1977) and Henssen \& Kantvilas (1985) respectively. Additional new taxa from NewZealand (Galloway 1983b, Martin 1962) also have particular relevance to Tasmania, as do the new species from the Australian mainland published by Archer (1980), Elix (1981a,b), Elix \& Johnston (1986), Elix \& Stevens (1979), Elix \& Armstrong (1983), Filson (1984) and Timdal (1984). New records for Tasmania are given by Bratt \& Cashlin (1975, 1976), Filson (1986, 1988b), Filson \& Rogers (1979), Elix \& Streimann (1982), Hertel (1987), Kantvilas (1985), Kantvilas \& James (1987 and in prep.), Kantvilas \& Jarman (1988), Kantvilas et al. (1985), Lumbsch (1988a) and Petersen \& Kantvilas (1986).

Although many of these developments have been incorporated by Filson (1983, 1986, 1988b) into his checklists of the Australian flora, there is considerable argument for maintaining the data for Tasmania separately. Firstly, Tasmania represents a distinct geographical entity and the phytogeographic affinities of its lichen flora are unique. These affinities are lost when subsumed within the data for a land mass as large and diverse as the Australian continent. Secondly, the increasing importance of lichens in the management and conservation of vegetation systems in Tasmania has generated considerable local demand for a checklist of the island's lichen flora.

It must be emphasised that the present paper does not represent a complete account of this flora. The state of knowledge within different genera and families remains patchy; hence the checklist is a blend of data from recent studies (see above) and information which has remained virtually unchanged since the list of Wetmore (1963), (e.g. in the genera Peltigera, Pertusaria, Physcia, Sticta, Usnea). Within these limitations, it represents a status quo report which will be updated as new information comes to hand.

\section{METHODS}

Names of all lichens previously reported from Tasmania, either in Wetmore (1963) or in subsequent papers (see above), are listed either in the checklist (if they are currently accepted) or in the list of excluded names. As far as practicable, original sources were checked in order to eliminate those records which have ariser through misinterpretation of the original reference. Names which are accepted but which are based solely on uncertain, mostly 19 th-century records are followed by a $(+)$. Taxa based on a Tasmanian type specimen are in bold type whilst taxa known only from the Bass Strait islands and not recorded from the Tasmanian mainland are listed in square brackets.

\section{RESULTS}

The present checklist contains 633 species plus 22 infraspecific taxa in 173 genera. Twenty-three taxa are known only from the Bass Strait islands. Fourteen species are recorded from Tasmania for the first time, based chiefly on the author's recent collections. These are: Arthroraphis citrinella, Cetraria australiensis, Coccotrema porinopsis, Hyperphyscia adglutinata, Lecanora blanda, Neofuscelia verrucella, Pertusaria superba, Phaeographis australiensis, Relicina subnigra, Rinodina bischoffii, Siphulastrum mamillatum, Sphaerophorus imshaugii, Stereocaulon gregarium and Usnea contexta. The checklist also includes some non-lichenised fungal genera which have been traditionally associated with the lichens, both taxonomically and ecologically, e.g. Chaenothecopsis, Mycocalicium and Lichenothelia. 


\section{CHECKLIST OF TASMANIAN LICHENS}

Acarospora citrina (Taylor) Zahlbr. ex Rech.

A. tasmanica Räsänen

Alectoria nigricans (Ach.) Nyl.



A. cinereopruinosa Schacrer s. lat

A. cinnabarina (DC.) Waltr. (+)

A. complanata Fée $(+)$

A. epipastoides Leighton (t)

A. miserula Nyl. (+)

A. tumidula (Ach.) Ach. (t)

Arthopyrenia sublitoralis (Leighton) Amold (+)

Arthothelium ilicinum (Taylor) P. James

A. interveniens (Nyl.) Zahlbr.

A. macrothecium (Fée) Massal. (+)

Arthroraphis alpina (Schaerer) R. Sant.

A. citrinella (Ach.) Poelt

\section{Austroblastenia parciseptata (Shirley) Sipman}

A. pupa Sipman

Bacidia buchananii (Stirton) Hellbom

$B$. leucocarpa Knight (t)

B. luteola (Schrader) Mudd (+)

B. melasemoides (Jatta) Zahlbr.

[B. millegrana (Taylor) Zahlbr. (+)]

B. otagoensis (Nyl.) Hellbom var. tasmanica (Jatta)

Zahlbr.

B. weymouthii (Shirley) Zahlbr.

Bactrospora dryina (Ach.) Massal.

Baeomyces absolutus Tuck.

$B$. arcuatus Stirton

B. heteromorphus Nyl. ex Church. Bab. \& Mitten

Bellemerea alpina (Sommerf.) Clauz. \& Roux

\section{Biatora byssacea Hampe}

Blastenia carnella (Nyl.) Müll. Arg. (+)

B. pulcherrima Müll. Arg.

[Buclia coniops (Wahlenb. ex Ach.) Th. Fr.] ( + )

$B$. disciformis (Fr.) Mudd (+)

B. levieri Jatta

[B. subalbula (Nyl.) Müll. Arg.] (+)

Calicium abietinum Pers.

C. adspersum Pers. ssp. australe Tibell

C. glaucellum Ach.

C. salicinum Pers.

C. trabinellum (Ach.) Ach.

C. tricolor F. Wilson
C. victorianum sep, (F. Wilson) Tibell victorianum

Caloplaca cerineila (Nyl.) Flagey (t)

C. chrysophthalma Degelius (t)

C. cinnabarina (Ach.) Zahlor. (t)

C. cirrina (Hoffm.) Wh. Fr. $(+)$

C. cribrosa (Mus) 2 ablbr.

C. fermghes (Huds.) me Fr. (-t)

C. flavonbescens (Huds.) bandon (t)

C. hotocarpa (Hoffon.) Wade (-t)

C. migulosa (Nyl.) Zahibr. (+)

C. saxicola (Hoffm.) Nordin (t)

C. sublobulata (Nyl.) Zahlbr. (+)

Candelariella reflexa (Nyl.) Letrau

Canoparmelia norpruinata Elix \& Johnston

Catapyrenium lachneum (Ach.) R. Sant.

Catillaria grossulina (Stirton) Zahlbr.

C. kelica (Stirton) Zahlbr.

C. tasmanica Räsänen

C. trachonoides (Nyl.) Zahlbr. (t)

C. umbratilis Jatta

Catinaria grossa (Pers. ex Nyl.) Vainio

C. laureri (Hepp ex Th. Fr.) Degelius

C. pulverea (Borrer) Vezda \& Poelt

Cetraria australiensis W. Weber ex Kärnef.

C. chlorophylla (Willd.) Vainio

C. islandica (L.) Ach. ssp. antarctica Kärnef.

Chaenotheca brachypoda (Ach.) Tibell

C. brunneola (Ach.) Müll. Arg.

C. carthusiae (Harm.) Lettau

C. chrysocephala (Turn. ex Ach.) Th. Fr.

C. ferruginea (Turn. ex Sm.) Migula

C. gracillima (Vainio) Tibell

C. hispidula (Ach.) Zahlbr.

C. stemonea (Ach.) Müll. Arg.

C. trichialis (Ach.) Th. Fr.

Chaenothecopsis debilis (Turn. \& Borrer ex Sm.) Tibell

C. hacmatopus Tibell

C. nigra Tibell

C. pusilla (Ach.) A. Schmidt

C. sagenidii Tibell

C. savonica (Räsänen) Tibell

C. tasmanica Tibell

C. viridireagens (Nádv.) A. Schmidt

Chiodecton colensoi (Massal.) Muill. Arg.

Chrysothrix candelaris (L.) Laundon

Cladia aggregata (Sw.) Nyl.

C. fuliginosa R. Filson 


\section{C. inflata (F. Wilson) D. Galloway \\ C. moniliformis Kantvilas \& Elix \\ C. retipora (Labill.) Nyl. \\ C. schizopora (Nyl.) Nyl. \\ C. sullivanii (Müll. Arg.) Martin}

Cladina confusa (R. Sant.) Follm. \& Ahti

C. mitis (Sandst.) Hustich

C. tasmanica (Ahti) Ahti

\section{Cladonia angustata Nyl}

C. bimberiensis Archer

C. capitellaia (J.D. Hook. \& Taylor) Church. Bab. var. capitellata

C. capitellaia var. interhiascens (Nyl.) Sandst.

C. capitellala var. squamatica Archer

C. cervicornis ssp. verticillata (Hoffm.) Ahti

C. chlorophaea (Flörke) Sprengel

C. coniodendroides F. Wilson (type material not located, species unknown)

C. corniculata Ahti \& Kashiwadani

C. cornuta (L.) Hoffm.

C. crispata (Ach.) Flotow

C. cryptochlorophaea Asahina

C. ecmocyna Leighton

C. fimbriata (L.) Fr.

C. floerkeana (Fr.) Flörke

C. furcata (Huds.) Schrader

C. gracilis (L.) Willd. ssp. tenerrima Ahti

C. humilis (With.) Laundon var. humilis

C. humilis var. bourgeanica Archer

C. krempelhuberi Vainio

C. kuringaiensis Archer

C. merochlorophaea Asahina

C. murrayi W. Martin

C. ochrochlora Flörke

C. paeminosa Archer

C. pertricosa Krempelh.

C. pleurota (Flörke) Schaerer

C. praetermissa Archer

C. pyxidata (L.) Hoffm.

C. ramulosa (With.) Laundon

C. rigida (J.D. Hook. \& Taylor) Hampe var. rigida

C. rigida var. acuta (Taylor) Archer ined.

C. sarmentosa (J.D. Hook. \& Taylor) Dodge

C. scabriuscula (Delise) Leighton

C. southlandica W. Martin

C. staufferi des Abb.

C. subdigitata Vainio

C. sulcata Archer var. sulcato

C. sulcata var. wilsonii (Archer) Archer

C. tessellata Ahti \& Kashiwadani

C. weymouthii F. Wilson ex Archer

Clathroporima eminentior (Nyl.) Müll. Arg. (+)

Cliostomum griffithii (Sm.) Coppins
Coccocarpia erythroxyli (Sprengel) Swinsc. \& Krog C. palmicola (Sprengel) Arvidsson \& D. Galloway

Coccotrema cucurbitula (Mont.) Müll. Arg.

C. porinopsis (Nyl.) Imshaug ex Yoshimura

Coelocaulon aculeatum (Schreber) Link

Coenogonium implexum $\mathrm{Nyl}$.

Collema coccophorum Tuck

C. crispum (Huds.) Weber

C. durietzii Degelius

C. fasciculare (L.) Wigg. var fasciculare

C. fasciculare var. colensoi Church. Bab.

C. flaccidum (Ach.) Ach.

C. glaucophthalmum Nyl. var. glaucophthalmum

C. glaucophthalmum var. implicatum (Nyl.) Degelius

C. laeve J.D. Hook. \& Taylor var. laeve

C. laeve var. senecionis (F. Wilson) Degelius

C. leucocarpum J.D. Hook. \& Taylor

C. quadriloculare $\mathrm{F}$. Wilson var. quadriloculare

C. quadriloculare var. tasmaniae $\mathrm{F}$. Wilson

C. subconveniens Nyl.

C. subflaccidum Degelius

\section{Conotremopsis weberiana Vezda}

Crocynia gossypina (Sw.) Massal. (+)

Cystocoleus niger (Huds.) Hariot

Degelia durietzii Arvidsson \& D. Galloway

D. gayana (Mont.) Arvidsson \& D. Galloway

Dendriscocaulon dendriothamnodes Dughi in D. Galloway

Dictyonema sericeum (Sw.) Berkley

Dimerella lutea (Dickson) Trevis

Diploicia canescens (Dickson) Massal.

Diploschistes muscorum (Scop.) R. Sant ssp. bartlettii Lumbsch

D. ocellatus (Vill.) Norman

D. scruposus (Schreber) Norman

D. sticticus (Körber) Müll. Arg.

[Diplotomma alboatrum (Hoffm.) Flotow (+)]

Dirinaria picta (Sw.) Clem. \& Shear

Ephebe fruticosa Henssen (type locality erroneously given as Grosse River, Tasmania by Henssen (1963); locality is in N.S.W.

E. lanata (L.) Vainio

Eremastrella crystallifera (Taylor) G. Schneider 
[Flavoparmelia euplecta (Stirton) Hale]

$F$. haysomii (Dodge) Hale

F. rutidota (J.D. Hook. \& Taylor) Hale

[F. soredians (Nyl.) Hale]

Fulgensia fulgens (Sw.) Elenkin (+)

F. subbracteata (Nyl.) Poelt $(t)$

Fuscidea absolodes (Nyl.) Hertel \& V, Wirth

Graphis angustata Eschw. (+)

G. insidiosa (Knight \& Mitten) J.D. Hook.

G. scripta (L.) Ach.

Gyalecta jenensis (Batsch) Zahlbr. (+)

Gyalidea hyalinescens (Nyl.) Vezda

Haematomma infuscum (Stirton) R.W. Rogers

$H$. sorediatum R.W. Rogers

Heterodea muelleri (Hampe) Nyl.

Heterodermia comosa (Eschw.) Follm. \& Redon (+)

[H. hypoleuca (Ach.) Trevis. (+)]

[H. japonica (Sato) Swinsc. \& Krog. (+)]

H. microphylla (Kurok.) Swinsc. \& Krog

H. obscurata (Ny1.) Trevis.

H. speciosa (Wulf.) Trevis. (+)

Hyperphyscia adglutinata (Flörke) Mayrhofer \& Poelt

Hypocenomyce australis Timdal

H. foveata Timdal

\section{Hypogymnia billardierei (Mont.) R. Filson}

$H$. enteromorphoides Elix

H. kosciuskoensis Elix

H. lugubris (Pers.) Krog var. lugubris

H. lugubris var. compactior (Zahlbr.) Elix

H. lugubris var, sublugubris (Müll. Arg.) Elix *

H. mundata (Nyl.) Rassad.

$H$. pulverata (Nyl.) Elix

H. subphysodes (Krempelh.) Filson var. subphysodes

$H$. subphysodes var. austerodioides Elix

H. tubularis (Taylor) Elix

H. turgidula (Bitter) Elix

Hypotrachyna laevigata (Sm.) Hale (+)

$H$. reducens (Nyl.) Hale

H. revoluta (Flörke) Hale

H. sinuosa (Sm.) Hale

\section{Knightiella splachnirima (J.D. Hook. \& Taylor) Gyelnik}

Laurera megasperma (Mont.) Zahlbr. var. tasmanica (Jatta) Zahlbr.

Lecanactis abietina (Ach.) Körber

L. subfarinosa (Knight) Hellbom (+)
Cecania vallatula Jatto

Lecanora atrella Jatta

L. blanda Nyl.

L. broccha Nyl.

L. caesiontubella Ach. $(+)$

L. carpinea (L.) Vainio (t)

L. cenisia Ach. (+)

L. lineolaia Müll. Arg. (+)

L. rupicola (L.) Zahlbr. (+)

L. subfusca (L.) Ach. (t)

[L. varia (Hoffm.) Ach. (+)]

Lecidea aniptiza Stirton I. intersociella Stirton

L. canorufescens Krempelh. $(+)$

L. cera-rufa (Shirley) Zahlbr.

L. contigua Fr. (+)

L. coromandelica Zahlbr.

L. elabens Fr. (+)

L. enteroleuca Ach. $(+)$

L. flindersii Crombie

L. hypersporella Stirton

L. hypnorum Libert

L. immarginata $\mathrm{R}$. Br. ex Crombie

L. laeta Stirton

L. lampra Stirton

L. leptocarpa Nyl. ex Church. Bab. \& Mitten

L. leptoloma Müll. Arg. $(+)$

L. minutula Müll. Arg. (+)

L. stuartii Hampe

L. subnexa Stirton

L. subtecta Stirton

L. turgidula Fr. (+)

Lecidella elaeochroma (Ach.) Choisy

Leioderma amphibolum (Knight) D. Galloway \& P.M. Jørg.

L. duplicatum (Müll. Arg.) D. Galloway \& P.M. Jørg.

L. pycnophorum $\mathrm{Nyl}$.

L. sorediatum D. Galloway \& P.M. Jørg.

Lepraria incana (L.) Ach.

L. lobificans $\mathrm{Nyl}$.

Leprocaulon arbuscula (Nyl.) Nyl.

L. microscopicum (Vill.) Gams ex D. Hawksw.

Leproloma membranacea (Dickson) Vainio

Leptogium biloculare $\mathrm{F}$. Wilson

L. coralloideum (May. \& Flot.) Vainio

L. cyanescens (Ach.) Körber

L. limbatum $\mathrm{F}$. Wilson

L. menziesii (Sm. ex Ach.) Mont.

L. pecten $\mathrm{F}$. Wilson

L. tasmanicum F.Wilson

L. victorianum $\mathrm{F}$. Wilson

Leptotrema lepadodes (Tuck.) Zahlbr. (+)

L. lepadodes var. endochrysoides (Jatta) Zahlbr. 


\section{[Lichenothelia solitarioides Henssen]}

Lichina coninis (O.F. Müll.) Agardh

L. tasmanica Henssen

Lobaria scrpbiculata (Scop.) DC.

Lopadium bfferum (Nyl.) Zahlbr. (+)

L. disciforme (Flotow) Poelt \& Vezda

L. heparicola Döbbeler, Poelt \& Vezda

Maronea constans (Nyl.) Hepp

Megaloblastenia marginiflexa (J.D. Hook. \& Taylor) Sipman

Megalospora biclipea (Shirley) Zahlbr. (type lost (Kantvilas 1988); best referred to Catinaria s. lat.

M. campylospora (Stirton) Sipman

M. gompholoma (Müll. Arg.) Sipman ssp. fuscolineata Sipman

M. lopadioides Sipman

M. subtuberculosa (Knight) Sipman

Melampilidium metabolum (Nyl.) Müll. Arg. (+)

Melanelia subglabra (Räsänen) Esslinger

Melaspilea circumserpens Nyl.

M. gemella (Eschw.) Nyl. (+)

Menegazzia aeneofusca (Müll. Arg.) R. Sant.

M. bullata (Stirton) Bitter

M. caesiopruinosa $\mathrm{P}$. James

$M$. caliginosa P. James \& D. Galloway

$M$. confusa $\mathrm{P}$. James

$M$. eperforata P. James \& D. Galloway

$M$. globulifera R. Sant.

$M$. inactiva $P$. James \& Kantvilas

M. minuta P. James \& Kantvilas

$M$. nothofagi (Zahlbr.) P. James \& D. Galloway

$M$. platytrema (Müll. Arg.) R. Sant.

$M$. retipora (Stirton) Bitter

M. subbullata P. James \& Kantvilas

$M$. subpertusa P. James \& D. Galloway

$M$. testacea P. James \& D. Galloway

$M$ ultralucens P. James \& D. Galloway

M. weindorferi (Zahlbr.) R. Sant.

Metus conglomeratus (F. Wilson) D. Galloway \& $\mathrm{P}$. James

Micarea prasina $\mathrm{Fr}$

M. prasinella (Jatta) M. Lamb

Microcalicium arenarium (Hampe ex Massal.) Tibell $M$. conversum Tibell

Microthelia analtiza (Stirton) Zahlbr. (+)

Miltidea ceroplasta (Church. Bab.) D. Galloway \& Hafellner
Multiclavula mucida (Pers.) R.H. Petersen

$M$. vernalis (Schw.) R.H. Petersen

Mycocalicium albonigrum (Nyl.) Tibel

M. subtile (Pers.) Szat.

$M$. victoriae (C. Knight ex F. Wilson) Tibell

Mycoporellum obscurum (Pers.) A.L. Smith (+)

[Neofuscelia glabrans (Nyl.) Esshinger]

$N$. imitatrix (Taylor) Esslinger

N. loxodella (Esslinger) Esslinger

N. pulla (Ach.) Esslinger

$N$. stygioides (Nyl. ex Crombie) Esslinger

$N$. verrucella (Esslinger) Esslinger

Neophyllis melacarpa (F. Wilson) F. Wilson

Nephroma australe Richard

N. cellulosum (Ach.) Ach. var. cellulosum

N. rufum (Church. Bab.) P. James

Normandina pulchella (Borrer) Nyl.

Ochrolechia frigida (Sw.) Lynge

O. pallescens (L.) Massal. (+)

O. parella (L.) Massal. (+)

o. weymouthii Jatta

\section{Opegrapha agelaeina Jatta}

O. stellata Knight

Pannaria fulvescens (Mont.) Nyl.

$P$. immixta Nyl

$P$. mariana (Fr.) Müll. Arg. $(+)$

$P$. pezizoides (Web.) Trevis.

$P$. rubiginosa (Thunb. ex Ach.) Delise (+)

Pannoparmelia angustata (Pers. in Gaud.) Zahlbr.

$P$. wilsonii (Räsänen) D. Galloway

Paraparmelia conranensis (Elix) Elix \& Johnston

$P$. lithophiloides (Kurok.) Elix \& Johnston

[P. mongaensis (Elix) Elix \& Johnston]

Parmelia cunninghamii Crombie

$P$. erumpens Kurok.

$P$. protosulcata Hale

P. pseudorelicina Jatta (type not located; unlikely to

belong to Parmelia s. strict.)

$P$. salcrambidiocarpa Hale

$P$. signifera $\mathrm{Nyl}$

$P$. sulcata Taylor

$P$. tenuirima J.D. Hook. \& Taylor

$P$. testacea Stirton

Parmeliella microphylla (Sw.) Müll. Arg. (+)

$P$. nigrocincta (Mont.) Müll. Arg.

$P$. rubiginascens (Nyl.) Müll. Arg. (+)

[P. subgranulata D. Galloway \& P.M. Jørg.] 
Parmelina labrosa (Zahlbr) Elix \& Johnston

$P$. stevensiana Elix \& Johnston

Parmelinopsis afrorevoluta (Krog \& Swinsc.) Elix \& Hale

$P$. horrescens (Taylor) Elix \& Hale ( + )

$P$. spumosa (Asahina) Elix \& Hale $(+)$

$P$. subfatiscens (Kurokawa) Elix \& Male

Parmentaria gregalis (Knight) Mull. Arg. (t)

Parmotrema chinense (Osbeck) Hale \& Ahti

$[P$. cristiferum (Taylor) Hale]

$P$. reticulatum (Taylor) Choisy

$P$. subcaperatum (Krempelh.) Hale (+)

Peltigera canina (L.) Willd. (t)

$P$. canina f. leucorrhiza (Flörke) Flörke $(+)$

$P$. degenii Gyelnik f. tasmaniae Gyelnik

$P$. didactyla (With.) Laundon

$P$. dolichorhiza (Nyl.) Nyl.

$P$. horizontalis (Huds.) Baumg. (+)

$P$. membranacea (Ach.) Nyl. $(+)$

$P$. polydactyla (Neck.) Hoffm. $(+)$

$P$. pusilla (Fr.) Körber $(+)$

$P$. rufescens (Weis) Humb. (+)

Peltula euploca (Ach.) Poelt ex Ozenda \& Clauz.

Pertusaria aggregata Müll. Arg. (+)

$P$. alpina Hepp ex Ahles

$P$. commutata Müll. Arg. (+)

$\boldsymbol{P}$. gibberosa Müll. Arg.

$P$. lacerans Müll. Arg. $(t)$

$P$. leucostoma (Bernh.) Mass. (+)

$P$. meridionalis Müll. Arg. var, xanthostoma Müll. Arg.

$(+)$

$P$. novaezelandiae Szat.

P. ornatula Müll. Arg. (+)

$P$ paratropa Müll. Arg. (+)

$P$. pertractata Stirton

$P$. superba Zahlbr.

$P$. truncata Krempelh.

$P$. tryptheliformis (Nyl.) (+)

Phaeographis australiensis Müll. Arg.

$P$. exaltata (Mont. \& v.d. Bosch) Müll. Arg.

Phaeophyscia endococcinoides (Poelt) Esslinger $P$. orbicularis (Neck.) Moberg

Phlyctis subuncinata Stirton

Phyllopsora congregans (Zahlbr.) D. Galloway

$P$. parvifolia (Pers.) Müll. Arg. (+)

Physcia adscendens (Fr.) Oliver

$P$. aipolia (Ehrh. ex Humb.) Fürnr. (+)

$[P$. alba (Fée) Müll. Arg. $(+)]$

$P$. albata (F. Wilson) Hale $(+)$

$[P$. albicans (Pers.) Thomson $(+)]$
$P$ caesia (Hotm) Purnr.

$P$. clementei (Sm.) lynge

$P$. stellaris (L.) Lynge

[P. tribacia (Ach) Nyl. (t)]

Physconia grisea (Lam.) Poelt (+)

Physma chilense Hue

Placopsis cribellans (Nyl) Rasanen

$P$. gelida (L..) Lindsay

$P$. parellina (Nyl.) Lamb

$P$. parellina f. microphylla Lamb

$P$. perrugosa (Nyl.) Nyl.

$P$. rhodocarpa (Nyl.) Wetmore

$P$. trachyderma (Krempelh.) P. James var. clavifera (Lamb) P. James

Placynthium nigrum (Huds.) S.F. Gray

Porina leptaleina (\$yl.) Müll. Arg.

Protoblastenia rupestris (Scop.) J. Steiner

Protoparmelia badia (Hoffm.) Hafellner (+)

$P$. petraeoides (Nyl. ex Hue) Hertel

Pseudephebe pubescens (L.) Choisy

Pseudocyphellaria ardesiaca D. Galloway

$P$. argyracea (Delise) Vainio

$P$. aurata (Sm.) Vainio

P. billardierei (Delise) Räsänen

$P$. colensoi (Church. Bab.) Vainio

$P$. coronata (Müll. Arg.) Malme

$P$. crocata (L.) Vainio

$P$. dissimilis (Nyl.) D. Galloway \& P. James

$P$. faveolata (Delise) Malme

P. glabra (J.D. Hook. \& Taylor) Dodge

$P$. granulata (Church. Bab.) Malme

$P$. insculpta (Stizenb.) D. Galloway

$P$. intricata (Delise) Vainio

P. multifida (Nyl.) D. Galloway \& P. James

$P$. neglecta (Müll. Arg.) Vainio

P. rubella (J.D. Hook. \& Taylor) D. Galloway \& P. James

Psilolechia clavulifera (Nyl.) Coppins

$P$. lucida (Ach.) Choisy

$P$. purpurascens Coppins \& Purvis

Psora decipiens (Hedw.) Hoffm. (+)

Psoroma allorhizum (Nyl.) Hue (t)

$P$. asperellum Nyl.

$P$. durietzii P. James \& Henssen

$P$. euphyllum Nyl.

$P$. hirsutulum Nyl. ex Crombie (+)

$P$. hypnorum (Vahl) S.F. Gray

$P$. leprolomum (Nyl.) Räsänen

P. microphyllizans (Nyl.) D. Galloway 
P. paleaceum (Fr.) $\mathrm{Nyl}$

$P$. pallidun Nyl. (+)

$P$. pholidotoides (Nyl.) Trevis.

$P$. pholido:um (Mont.) Müll. Arg. (+)

P. soccatum R.Br.

$P$. sphinctrinum (Mont.) Nyl. (+)

$P$. xanthomelanum Nyl. $(+)$

Psoromidium aleuroides (Stirton) D. Galloway

$P$. versicolor (J.D. Hook. \& Taylor) D. Galloway

Punctelia borreri (Sm.) Krog

P. subflava (Taylor) Elix \& Johnston

$P$. subrudecta (Nyl.) Krog

Pycnothelia caliginosa D. Galloway \& P. James

\section{Pyrenopsis tasmanica Nyl.}

\section{Pyrenula chloroplaca Shirley}

$P$. galactina (Shirley) Kantvilas

$P$. kunthii (Fée) Fée (+)

Pyrgillocarpon indicum (Krempelh.) Nádv. in Tibell

Pyxine nubila Moberg

Ramalea cochleata Müll. Arg.

Ramalina caespitella N. Stevens

[R. canariensis Steiner]

R. celastri (Sprengel) Krog \& Swinsc. ssp. celastri

R. celastri ssp. ovalis (J.D. Hook. \& Taylor) N. Stevens

$R$. fimbriata Krog \& Swinsc.

R. fissa (Müll. Arg.) Vainio

$R$. glaucescens Krempelh.

R. inflata (J.D. Hook. \& Taylor) J.D. Hook. \& Taylor ssp. inflata

$R$. inflata ssp. australis N. Stevens

$R$. unilateralis $\mathrm{F}$. Wilson

R. whinrayi N. Stevens

Ramalodium succulentum $\mathrm{Nyl}$.

Relicina limbata (Laurer) Hale

$R$. subnigra Elix \& Johnson

Rhizocarpon badioatrum (Flörke ex Sprengel) Th.Fr. f. atroalbum (L.) Malme (+)

$R$. geographicum (L.) DC.

$R$. petraeum (Wulfen) Massal. (+)

\section{Rinodina asperata (Shirley) Kantvilas}

$R$. bischoffii (Hepp) Massal.

\section{$\boldsymbol{R}$. brattii Mayrhofer}

$R$. conradii Körber

R. dissa (Stirton) Mayrhofer

$R$. exigua (Ach.) S.F. Gray (+)

R. gennarii Bagl.

R. murrayi Mayrhofer

$R$. occulta (Körber) Sheard
R. peloleuca (Nyl.) Müll. Arg.

$R$. procellarum (Massal.) Mayrhofer

$R$. subcrustacea (Müll. Arg.) Zahlbr

$\boldsymbol{R}$. tasmanica Mayrhofer

R. thiomela (Nyl.) Muill. Arg.

\section{[Roccellina expectata Tehler]}

Roccellinastrum neglectum Henssen \&Vobis

Sagenidium molle Stirton

Sarrameana tasmanica Vezda \& Kantvilas

Schismatomma congerens (Nyl.) Zahlbr.

Scoliciosporum pruinosum (P. James) Vezda

Siphula complanata (J.D. Hook. \& Taylor) R. Sant.

S. decumbens Nyl.

S. foliacea D. Galloway

S. fragilis (J.D. Hook. \& Taylor) J.Murray

S. jamesii Kantvilas

Siphulastrum mamillatum (J.D. Hook. \& Taylor) D. Galloway

S. triste Müll. Arg.

Sphaerophorus imshaugii Ohlsson in D. Galloway

$S$. insignis Laurer

S. macrocarpus Ohlsson in D. Galloway

$S$. melanocarpus (Sw.) DC.

$S$. murrayi Ohlsson in Tibell

S. notatus Tibell

$S$. patagonicus (Dodge) Ohlsson in D. Galloway

$S$. ramulifer Lamb

S. scrobiculatus (Church. Bab.) Sato

S. tener Laurer

Sphinctrina leucopoda Nyl.

S. tubaeformis Massal.

Stephanocyclos henssenianus Hertel

Stereocaulon caespitosum Redinger

S. corticatulum Nyl. var. corticulatum

S. corticatulum var. humile (Müll. Arg.) Lamb

S. corticatulum var. subcorticatum (Räsänen) Lamb

S. gregarium Redinger

S. ramulosum ( $\mathrm{Sw}$.) Räuschel

S. trachyphloeum Lamb

$S$ vesuvianum Pers.

Sticta damaecornis (Sw.) Ach. (+)

S. fuliginosa (Dickson) Ach.

S. limbata (Sm.) Ach.

S. macrophylla Delise $(+)$

S. querzicans Delise (+)

S. stipitata Knight

S. subcaperata (Nyl.) Nyl. (+)

S. sublimbata (Steiner) Swinsc. \& Krog 
S. sylvatica (Huds.) Ach. (t)

S. weigelii (Ach.) Vainio (t)

Synalissa cancellata F. Wilson (+)

Teloschistes chrysophthalmus (L.) Th. Fr.

T. flavicans ( $\mathrm{Sw}$ ) Norm.

$T$. sieberianus (Laurer) Hillm.

T. spinosus (J.D. Hook. \& Taylor) I. Murray

$T$. spinosus f. subteres R. Filson

$T$. velifer $\mathrm{F}$. Wilson

[T. velifer f. nodulosa (J. Murray) R. Filson]

Tephromela atra (Huds.) Hafellner

Thamnolia vermicularis (Sw.) Ach. ex Schaerer var. vermicularis

T. vermicularis var. subuliformis (Ehrh.) Schaerer

Thelotrema decorticans Müll. Arg.

T. lepadinum (Ach.) Ach.

T. subdenticulatum (Zahlbr.) G. Salisb.

T. subgranulosum Jatta

Thysanothecium hookeri Mont. \& Berk.

T. scutellatum (Fr.) D. Galloway

Toninia bullata (Meyen \& Flotow) Zahlbr.

T. microlepis (Müll. Arg.) Zahlbr. (+)

Trapelia coarctata (Turner) Choisy (+)

Trapeliopsis colensoi (Church. Bab.) G. Schneider

T. granulosa (Hoffm.) H.T. Lumbsh

Tremolecia atrata (Ach.) Hertel

Turgidosculum complicatulum (Nyl.) J. Kohlmeyer \& E. Kohlmeyer

Umbilicaria cylindrica (L.) Delise ex Duby

$U$. hyperborea (Ach.) Hoffm.

$U$. polyphylla (L.) Baumg.

$U$. propagulifera (Vainio) Llano

U. subglabra (Nyl.) Harm.

Usnea acromelana Stirton

$U$. angulata Ach. (+)

$U$. baileyi (Stirton) Zahibr.

$U$. capillacea Motyka

$U$. ceratina Ach. (t)

$U$. contexta Motyka

$U$. hirta (Müll. Arg.) Weber em. Motyka (+)

$U$. inermis Motyka

$U$. leprosa Motyka

$U$. maculata Stirton

U. molliuscula Stirton

$U$. ramulosissima Stevens \& Rogers

$U$. rubicunda Stirton

$U$. scabrida Taylor

$U$. spilota Stirton (+)

U. subcapillaris (D. Galloway) F.J. Watker
U. porquescens Stirton

U. torulosa (Müll. Arg.) Zahlbr.

U. trichodea Ach. (+)

U. xanthopoga Nyl. (+)

Verrucaria maura Wahlenb. ex Ach.

V. microsporoides Nyl.

Verdaét obscura Döbbeler

\section{Wawea Truliculosa Henssen Kanturlds}

Xanthopamelia adhaerens (Nyl.) Hale

$X$. alexandrensis Elix \& Johnston

$X$. amphixantha (Muill. Arg,) Hale

$X$ amplexula (Stirton) Elix \& Johnston

$X$. antleriformis (Elix) Elix \& Johnston

$X$. arapilensis (Elix \& $\mathrm{P}$. Armstr.) Filson

$X$. australasica D. Galloway

$X$. bungendorensis (Elix) Elix \& Johnston

$X$. cheelii (Gyelnik) Hale

$X$. congesta (Kurok. \& Filson) Elix \& Johnston

$X$. constrictans (Nyl. ex Crombie) Hale

$X$. cordillerana (Gyelnik) Hale

$X$. dichotoma (Müll. Arg.) Hale

$X$. digitiformis (Elix \& P. Armstr.) Filson

$X$. dissitifolia Kurok. ex Elix \& Johnston

$X$. elixii Filson

$X$. filarszkyana (Gyelnik) Hale

$X$. flaviscentireagens (Gyelnik) D. Galloway

X. furcata (Müll. Arg.) Hale

X. glareosa (Kurok. \& Filson) Elix \& Johnston

$X$. hybridiza Elix \& Johnston

[X. incerta (Kurok. \& Filson) Elix \& Johnston]

$X$. isidiigera (Müll. Arg.) Elix \& Johnston

$X$. isidiosa (Müll. Arg.) Elix \& Johnston

[X. lineola (Berry) Hale]

$X$. mannumensis (Elix) Elix \& Johnston

$X$. metaclystoides (Kurok. \& Filson) Elix \& Johnston

$X$. mexicana (Gyelnik) Hale

$X$. mougeotina (Nyl.) D. Galloway

X. multipartita (R. Br.) Hale

$X$. neotinctina (Elix) Elix \& Johnston

$X$. notata (Kurok.) Hale

$X$. phillipsiana (Filson) Elix \& Johnston

$X$. pseudohypoleia (Elix) Elix \& Johnston

X. pustuliza (Elix) Elix \& Johnston

[X. remanens (Elix) Elix \& Johnston]

$X$. scabrosa (Taylor) Hale

X. streimannii (Elix \& P. Armstr.) Elix \& Johnston

$X$. subnuda (Kurok.) Hale

$X$. substrigosa (Hale) Hale

$X$. taractica (Kremp.) Hale

$X$. tasmanica (J.D. Hook. \& Taylor) Hale

$X$. tegeta Elix \& Johnston

$X$. thamnoides (Kurok.) Hale

$X$. verdonii Elix \& Johnston

$X$. vicaria Elix \& Johnston

$X$. willisii (Kurok. \& Filson) Elix \& Johnston

Xanthoria ligulata (Körber) P. James

$X$. parietina (L.) Th. Fr. 


\section{DELETED NAMES}

Acarospora schleicheri (Ach.) Massal. Probably refers to A. citrina (Tayl.) Zahlbr. ex Rech. (Lumbsch 1988b) Alectoria lanata (Neck.) Nyl. $\equiv$ Pseudephebe pubescens (L.) Choisy

Alectoria pubescens (L.) Howe $\equiv$ Pseudephebe pubescen (L.) Choisy

Anaptychia spp. All Tasmanian records of this genus refer to species of Heterodermia.

Anzia angustata (Pers. in Gaud.) Müll. Arg. = Pannoparmelia angustata (Pers. in Gaud.) Zahlbr.

Anzia colpodes (Ach.) Stizenb. Misapplied to Pannoparmelia angustata (Pers. in Gaud.) Zahlbr.

Anzia wilsonii Räsänen $\equiv$ Pannoparmelia wilsonii (Räsänen) D. Galloway

Arthonëa multiformis Shirley $\equiv$ Arthothelium ilicinum (Taylor) P. James

Arthothelium ferax (Müll. Arg.) $\equiv$ A. interveniens (Nyl.) Zahibr.

Arthothelium infuscatum (Krempelh.) Müll. Arg. $\equiv A$. interveniens (Nyl.) Zahlbr.

Aspicilia alpina (Sommerf.) Arnold $\equiv$ Bellemerea alpina (Sommerf.) Clauz. \& Roux

Baeomyces fungoides (Sw.) Ach. Refers to B. arcuatus Stirton in Australasia (see Kantvilas \& James 1987).

Baeomyces ramalinellus Nyl. Misapplied to $B$. arcuatus Stirton.

Baeomyces roseus Pers. Not present in Tasmania; most likely misapplied to $B$. arcuatus Stirton.

Baeomyces squamarioides Nyl. = Knightiella splachnirima (J.D. Hook. \& Taylor) Gyelnik

Blastenia consanguinea Müll. Arg. Misidentification of Megaloblastenia marginiflexa. (J.D. Hook. \& Taylor) Sipman (Kantvilas 1988).

Blastenia endochromoides Müll. Arg. 三 Megaloblastenia marginiflexa (J.D. Hook. \& Taylor) Sipman

Bombyliospora tuberculosa (Fée) Massal. (三 Megalospora tuberculosa (Fée) Sipman). Not present in Tasmania (Sipman 1983).

Buellia dissa Stirton $\equiv$ Rinodina dissa (Stirton) Mayrhofer

Buellia parasema (Ach.) De Not. $\equiv B$. disciformis (Fr.) Mudd

Buellia polospora (Leighton) Shirley var. asperata Shirley $\equiv$ Rinodina asperata (Shirley) Kantvilas

Buellia pulchella (Schrad.) Tuck. 三 Catolechia wahlenbergii (Ach.) Körber. Not present in Australia or Tasmania (R.W. Rogers, pers. comm.)

Calicium lenticulare (Hoffm.) Ach. Not present in Tasmania (Tibell 1987).

Calicium piperatum $\mathrm{F}$.Wilson $\equiv C$. victorianum ssp. victorianum ( $\mathrm{F}$. Wilson) Tibell

Calicium quercinum Pers, var. microcarpum F.Wilson $\equiv$ C. glaucellum Ach.

Calicium robustellum Nyl. Not recorded for Tasmania by Tibell (1987).

Calicium roscidulum Nyl. ex $\mathrm{F}$. Wilson $\equiv C$. trabinellum (Ach.) Ach.

Calicium roseo-albidum $\mathrm{F}$. Wilson $\equiv$ C. salicinum Pers.
Calicium victoriae $\mathrm{F}$. Wilson $\equiv$ Mycocalicium victoriae (F. Wilson) Tibel]

Caloplaca aurantiaca (Lightf.) Th. Fr. $\equiv C$. flavorubescens (Huds.) Laundon

Canoparmelia norsticticata (Ștevens) Elix \& Hale. Not recorded for Tasmania by Elix \& Stevens (1979).

Carbonea phaeostoma (Nyl.) Hertel. Not recorded for Tasmania by Hertel (1984).

Catillaria melanotropa (Nyl.) Zahlbr. The specimen upon which this record is based (Shirley 1894) is C. tasmanica Räsănen (see Kantvilas 1988).

Catinaria versicolor (Fée) Sipman. Although listed for Tasmania by Filson (1988b), this name appears to have been widely misapplied by early workers to several species of Megalospora or Catinaria.

Catolechia wahlenbergii (Ach.) Körber. Not present in Australia (R. Rogers, pers. comm.).

Cetraria scutata (Wulf.) Poetsch $\equiv C$. chlorophylla (Willd.) Vainio

Chiodecton perplexum Nyl. The specimen upon which the Tasmania record is based (Shirley 1894) is of C. colensoi (Massal.) Müll. Arg. (see Kantvilas 1988).

Cladina alpestris (L.) Nyl. Not present in Tasmania (Ahti 1984).

Cladina leptoclada (des Abb.) D. Galloway $\equiv C$. confusa (R. Sant.) Follm. \& Ahti

Cladina rangiferina (L.) Nyl. Not present in Tasmania (Ahti 1984).

Cladina sylvatica (Hofm.) Leighton. Not present in Tasmania (Ahti 1984).

Cladonia acuminata (Ach.) Norrl. Not recorded for Australia by Archer (in prep.).

Cladonia aggregata (Sw.) Sprengel $\equiv$ Cladia aggregata (Sw.) Nyl.

Cladonia aggregata f. subdivergens Hellbom $\equiv$ Cladia aggregata (Sw.) Nyl.

Cladonia aggregata var. tenera F. Wilson $\equiv$ Cladia aggregata (Sw.) Nyl.

Cladonia amaurocraea (Florke) Schaerer. An arctic and subarctic Northern Hemisphere species, not recorded for Australia by Archer (in prep.).

Cladonia anomaea (Ach.) Ahti \& P. James $\equiv C$. ramulosa (With.) Laundon

Cladonia bacillaris (Ach.) Nyl. Included in C. macilenta Hoffm. (Christensen 1987) and not recorded for Tasmania by Archer (in prep.)

Cladonia borbonica (Delise) Nyl. Not recorded for Australia by Archer (in prep.).

Cladonia calycantha Delise ex Nyl. Not present in Tasmania. Probably refers to $C$. cervicornis ssp. verticillata (Hoffm.) Ahti (see Ahti 1983).

Cladonia campbelliana (Vainio)Gyelnik $\equiv C$. sarmentosa (J.D. Hook. \& Taylor) Dodge

Cladonia capitata (Michaux) Sprengel. Refers to $C$. tessellata Ahti \& Kashiwadani in Tasmania.

Cladonia carassensis Vainio. This species is presently considered to be a thamnolic acid-containing form of. C. crispata (Ach.) Flotow (Stenroos 1988). 
Cladonia cariosa (Ach.) Sprengel. Refers to C. tessellata Ahti \& Kashiwadani in Tasmania.

Cladonia carneopallida (Flörke) Laurer. Not recorded for Australia by Archer (in prep.).

Cladonia cenotea (Ach.) Schaerer. Not recorded for Australia by Archer (in prep.).

Cladonia ceratophylla (Sw.) Sprengel. Refers to $C$. praetermissa Archer (Filson 1986).

Cladonia coccifera. (L.) Willd. Not present in Tasmania (Archer 1988a).

Cladonia coniocraea auct. Does not appear to occur in Tasmania (Archer, pers. comm.).

Cladonia conoidea Ahti $\equiv$ C.humilis (With.) Laundon.

Cladonia conisia auct. $\equiv C$. humilis var. bourgeanica (Archer 1988b).

Cladonia cornicularia Laurer $\equiv$ Cladia aggregata (Sw.) Nyl.

Cladonia decorticata (Flörke) Sprengel. Not recorded for Australia by Archer (in prep.)

Cladonia decurva Taylor ex Church. Bab. \& Mitten $\equiv$ C. scabriuscula (Delise) Nyl. (Archer, pers. comm.).

Cladonia deformis (L.) Hoffm. All records of this species from Tasmania appear to refer to $C$. pleurota (Flörke) Schaerer.

Cladonia digitata (L.) Hoffm. Not recorded for Australia (Archer 1988a).

Cladonia enantia Nyl. Not present in Tasmania. All records probably refer to $C$. tessellata Ahti \& Kashiwadani.

Cladonia farinacea (Vainio) Evans. Not recorded for Australia by Archer (in prep.).

Cladonia firma (Laurer) Krempelh. $\equiv C$. murrayi Martin.

Cladonia foliacea (Huds.) Willd. Not recorded for Australia by Archer (in prep.).

Cladonia gorgonina. (Bory) Vainio $\equiv$ Cladia aggregata (Sw.) Nyl.

Cladonia impexa Harm. [ $=$ Cladina portentosa (Duf.) Follm.]. Not present in Tasmania.

Cladonia macilenta Hoffm. Not present in Tasmania.

Cladonia neozelandica Vainio. Not present in Tasmania.

Cladonia parasitica (Hoffm.) Hoffm. Not recorded for Australia by Archer (in prep.).

Cladonia phyllophora Hoffm. Not recorded for Australia by Archer (in prep.).

Cladonia pityrea (Flörke) Fr. $\equiv$ C. ramulosa (With.) Laundon.

Cladonia polydactyla (Flörke) Nyl. Not recorded for Australia by Archer (in prep.).

Cladonia portentosa (Duf.) Coem. Not present in Tasmania and referrable to Cladina.

Cladonia rangiformis Hoffm. Not present in Tasmania.

Cladonia rappii var. exilior (des. Abb.) Ahti $\equiv C$. cervicornis ssp. verticillata (Hoffm.) Ahti.

Cladonia rei Schaerer. Not present in Tasmania. Record cited by Kantvilas \& Jarman (1988) refers to material of C. ramulosa (With.) Laundon containing homosekikaic acid.

Cladonia sparassa (Ach.) Hampe. $\equiv$ C. squamosa (Scop.) Hoffm. Not recorded for Australia by Archer (in prep.).

Cladonia squamosa (Scop.) Hoffm. Not recorded for Australia by Archer (in prep.).
Cladonia squamosula Müll. Arg. var. squamosula $\equiv$ C. rigida var. rigida (J.D. Hook. \& Taylor) Hampe.

Cladonia squamosula var. subsquamosula Archer $\equiv$ $C$. rigida var. acuta (Taylor) Archer.

Cladonia subcervicornis (Vainio) Kemst. Not recorded for Australia by Archer (in prep.).

Cladonia subsquamosa (Nyl.) Vainio. Not present in Tasmania (Archer, in prep.).

Cladonia subsubulata Nyl. This species is presently considered to be a thamnolic-acid-containing form of C. crispara (Ach.) Flowtow (Stenroos 1988).

Cladonia subulata (L.) Wigg. Refers to C. conniculata Ahti \& Kashiwadani in Tasmania.

Cladonia terebrata (Laurer) Flörke $\equiv$ Cladia aggregata (Sw.) Nyl

Cladonia uncialis (L.) Wigg. Not present in Tasmania. Probably a misidentification of $C$. capitellata (J.D. Hook. \& Taylor) Church. Bab.

Cladonia verticillata (Hoffm.) Schaerer $\equiv C$. cervicornis ssp. verticillata (Hoffm.) Aht

Cladonia wilsonit Archer $\equiv \mathrm{C}$. sulcata var. wilsonii (Archer) Archer

Coccocarpia cronia (Tuck.) Vainio $\equiv C$. palmicola (Spreng.) Arvidsson \& D. Galloway

Coccocarpia gayana (Mont.) Nyl. E Degelia gayana (Mont.) Arvidsson \& D. Galloway

Coccocarpia parmelioides (Hook.) Tuck $\equiv C$. erythroxli (Spreng.) Swinsc. \& Krog.

Coenogonium acrocephalum Mïll. Arg. Misidentification of $C$. implexum Nyl. (see Kantvilas 1988).

Coenogonium linkii Ehrh. Not in Tasmania and probably misapplied to $C$. implexum Nyl.

Coenogonium rigidulum Müll. Arg. Not in Tasmania and probably misapplied to $C$. implexum Nyl.

Collema nigrescens (Huds.) DC. Misapplied to C.glaucophthalmum Nyl. (Degelius 1974).

Collema robillardii (Müll. Arg.) Stizenb. Misapplied to C. quadriloculare F. Wilson (Degelius 1974).

Collema thysaneum Ach. Not in Tasmania and probably misapplied to both $C$. leucocarpum J.D. Hook. \& Taylor and $\mathcal{C}$. fasciculare (L.) Wigg. (Degelius 1974).

Cornicularia aculeata (Schreber) Ach. $\equiv$ Coelocaulon aculeatum (Schreber) Link.

Crocynia mollescens Nyl. Most probably refers to a species of Lepraria.

Cyphelium emergens (F. Wilson) Zahlbr. E Trachylia emergens $\mathrm{F}$. Wilson. This species represents a nonlichenised fungus (Tibell 1987).

Cystocoleus ebeneus (Dillwyn) Thwaites $\equiv C$. niger (Hudson) Hariot

Delisea pseudosticta Fée $\equiv$ Pseudocyphellaria glabra (J.D. Hook. \& Taylor) Dodge.

Dermatocarpon hepaticum (Lam.) Th.Fr. $\equiv$ Catapyrenium lachneum (Ach.) R. Sant.

Diploschistes euganeus (Massal.) Steiner. Not present in Tasmania (H.T. Lumbsch, pers. comm.).

Diploschistes subocellatus (Nyl.) Zahlbr。 $\equiv$ D. ocellatus (Vill.) Norm. (H.T. Lumbsch, pers. comm.).

Dufourea collodes J.D. Hook. \& Taylor $\equiv$ Cladia aggregata (Sw.) Nyl. 
Ephebe pubescens Fr. Wilson's (1893) record of this taxon refers to Pseudephebe pubescens (L.) Choisy.

Ephebe tosmanica Crombie. Known only from the type collection (Grosse River, N.S.W.). This locality is frequenty quoted as being in Tasmania due to an error by Crombie (1880).

Flavoparmelia ferax (Müll. Arg.) Hale. Not known from Tasmania or the Bass Strait islands (J. Elix, pers. comm.)

Gomphillus baeomyceoides Wilson $\equiv$ Bacidia buchananii (Stirton) Hellbom

Graphis intricata Fée. Incorrectly listed for Tasmania by Shirley (1893) on the basis of a collection by Robert Brown at Grosse River, N.S.W. (see Crombie 1880).

Gyalecta cupularis (Hedwig) Schaerer $\equiv G$. jenensis (Batsch) Zahlbr.

Gymnoderma melacarpum (F. Wilson) Yoshim. $\equiv$ Neophyllis melacarpa (F. Wilson) F. Wilson.

Heterodermia pseudospeciosa (Kurok.) Culb. Erroneously reported by Kantvilas et al. (1985) on the basis of a misidentification of $H$. microphylla (Kurok.) Swinsc. \& Krog (see Kantvilas \& James 1987).

Heterodermia tremulans (Müll. Arg.) Culb. $\equiv$ H. speciosa (Wulf.) Trevis.

Heterothecium pauciseptatum Shirley $\equiv$ Austroblastenia pauciseptata (Shirley) Sipman

Hypogymnia encausta (Sm.) W. Watson. Refers to H. tubularis (Taylor) Elix (Filson 1986).

Hypogymnia enteromorpha (Ach.) Nyl. Refers to H. enteromorphoides Elix (Filson 1986) in Australia.

Hypogymnia physodes (L.) Nyl. Widely misapplied to several species of Hypogymnia in Tasmania, especially to $H$. subphysodes (Krempelh.) Filson.

Hypogymnia vittata (Ach.) Gasilien. Not present in Tasmania (Elix 1979).

Lecanora angulosa (Schreber) Ach. $\equiv L$. carpinea (L.) Vainio

Lecanora atra (Huds.) Ach. $\equiv$ Tephromela atra (Huds.) Hafellner

Lecanora badia (Hoffm.) Ach. = Protoparmelia badia (Hoffm.) Hafellner

Lecanora coarctata $(\mathrm{Sm}$.) Ach. $\equiv$ Trapelia coarctata (Sm.) Choisy

Lecanora glaucoma (Hoffm.) Ach. $\equiv$ L. rupicola (L.) Zahlbr

Lecanora parmelina Zahlbr. $\equiv$ L. broccha $\mathrm{Nyl}$.

Lecanora sordida (Pers.) Th.Fr. $\equiv$ L. rupicola (L.) Zahlbr.

Lecanora sulphurea (Hoffm.) Ach. Origin of record doubtful and no Tasmanian specimens identified as this species have been seen.

Lecidea cinnabarina Sommerf. Refers to L. laeta Stirton

Lecidea crystallifera Taylor $\equiv$ Eremastrella crystallifera (Taylor) G. Schneider

Lecidea decipiens (Hedw.) Ach. $\equiv$ Psora decipiens (Hedw.) Hoffm.

Lecidea dicksonii auct. $\equiv$ Tremolecia atrata (Ach.) Hertel Lecidea friesii Ach. IE Hypocenomyce friesii (Ach.) P. James \& G. Schneider]. Not present in Tasmania. Lecidea interversa Nyl. $\equiv L$. canorufescens Krempelh.

Lecidea ostreata (Hoffm.) Schaerer. Refers to a species of Hypocenomyce.
Lecidea petraeoides Nyl. ex Hue $\equiv$ Protoparmelia petraeoides (Nyl. ex Hue) Hertel

Lecidea russula Ach. Refers to $L$. laeta Stirton in Tasmania.

Lepraria candelaris (L.) Fr. $\equiv$ Chrysothrix candelaris (L.) Laundon

Leptogium azureum (Sw.) Mont. in Webb \& Berthelot Not in Tasmania. Most material given this name in Australia is Collema subconveniens Nyl. (D. Verdon, pers. comm.).

Leptogium brebissonii Mont. in Webb \& Berthelot. Not in Tasmania. Easily confused with small Collema spp. (D. Verdon, pers. comm.).

Leptogium chloromelum var. granulare Müll. Arg. The specimen upon which this record is based (Shirley 1894) is referrable to Psoroma hypnorum (Vahl.) S.F. Gray (Kantvilas 1988).

Leptogium rugosum Sierk. Not present in Tasmania.

Leptogium scotinum (Ach.) Fr. Not present in Tasmania.

Leptogium tenuissimum (Dickson) Fr. The sole Tasmanian record of this species is attributed to Hampe (1852) by Wetmore (1963), but the original reference is to Parmelia tenuissima ( $\equiv P$. signifera) and not to a Leptogium.

Leptogium tremelloides (Linn. f.) S.F. Gray. Widely used name in early literature, apparently misapplied to $L$. azureum (Sw.) Mont. and L. cyanescens (Ach.) Körber.

Letrouitia domingensis (Pers.) Hafellner \& Bellemère. A tropical species not recorded for Tasmania by Hafellner (1981).

Lichina pygmaea (Lightf.) Agardh var, intermedia Church. Bab. $\equiv$ L. confinis (O.F. Mull.) Agardh

Lobaria crenulata (J.D. Hook.) Trevis. This record is based on "a single specimen of poor quality" (Babington \& Mitten 1859) collected by R.C. Gunn The specimen is cited in the catalogues of Müller (1887), Shirley (1893) and Wilson (1893), each time as a different Lobaria species, and thus has led to the introduction of several Lobaria names into Tasmanian (and Australian) checklists. Given the dubiousness of the original identification, and the lack of more recent gatherings, $L$. crenulata and the other species names associated with Gunn's specimen (see below) are deleted.

Lobaria herbacea (Huds.) DC. See under $L$. crenulata (above).

Lobaria laetevirens (Lightf.) Zahlbr. See under $L$. crenulata (above).

Lobaria leucocarpa (Müll. Arg.) Zahlbr. झ Knightiella splachnirima (J.D. Hook. \& Taylor) Gyelnik

Lobaria patinifera (Taylor) Hue. See under $L$. crenulata (above).

Lobaria sublaevis Nyl. See under $L$. crenulata (above).

Lopadium pauciseptatum (Shirley) Zahlbr. \# Austroblastenia pauciseptata (Shirley) Sipman

Mastodia tessellata auct. $\equiv$ Turgidosculum complicatulum (Nyl.) J. Kohlmeyer \& E. Kohlmeyer Megalospora coccodes (Bel.) Sipman. Not recorded for Tasmania by Sipman (1983).

Megalospora sulphurata var. nigricans (Müll. Arg.) Riddle. Not present in Tasmania (Sipman 1983). 
Megalospora tasmanica (Jatta) Zahlbr. Sipman (1983) observed that the type specimen has acicular spores and is therefore not a Megalospora.

Megalospora taylori Dodge. Not accepted by Sipman (1983).

Menegazzia diatrypa (Ach.) Massal. $[\equiv M$. terebrata (Hoffm.) Massal.]. Not present in Tasmania.

Menegazzia myriotrema (Müll. Arg.) R. Sant. $\equiv M$. platytrema (Müll. Arg.) R. Sant. (P. James, pers. comm.).

Menegazzia pertransita (Stirton) R. Sant. Refers to $M$. weindorferi (Zahlbr.) R. Sant. in Tasmania.

Menegazzia pertusa (Schrank) B. Stein $[=M$. terebrata (Hoffm.) Massal.] Not present in Tasmania.

Nephroma antarcticum (Wulf.) Nyl. Refers to N. australe A. Rich.

Nephroma helveticum Ach. Not present in Tasmania (White \& James 1988).

Nephroma lepidophyllum Räsänen ex Gyelnik $\equiv N$. cellulosum var. isidioferum J, Murray. Not present in Tasmania (White \& James 1988).

Nephroma resupinatum (L.) Ach. f. papyraceum (Hoffm.) Ach. $\equiv N$. helveticum Ach. Not present in Tasmania.

Neuropogon acromelanus var. decipiens Lamb $\equiv$ Usnea acromelana Stirton.

Neuropogon acromelanus var. inactivus Lamb $\equiv$ Usnea torulosa (Müll. Arg.) Zahlbr.

Neuropogon antarcticus (Du Rietz) Lamb $\equiv$ Usnea antarctica Du Reitz). Not present in Tasmania (Walker 1985).

Neuropogon ciliatus (Nyl.) Krempelh. [ (Nyl.) Du Rietz]. Occurrence in Tasmania doubtful (Walker 1985).

Neuropogon melaxanthus (Ach.) Nyl. [三 Usnea aurantiaco-atra (Jacqu.) Bory]. Not present in Tasmania (Walker 1985).

Neuropogon sulphureus (Th. Fr.) Hellbom (三Usnea sphacelata R. Br.) Not present in Tasmania (Walker 1985).

Ochrolechia pseudotartarea (Vainio) Verseghy. Record doubtful.

Pannaria imbricata $\mathrm{Nyl}=$ Psoroma asperellum Nyl.

Parmelia adpressa Krempelh. (三 Xanthoparmelia succedans Elix \& Johnston). Not present in Tasmania (Elix et al. 1986).

Parmelia alpicola Th. Fr. Not present in Tasmania. Possibly misapplied to Neofuscelia stygioides (Nyl. ex Crombie) Esslinger.

Parmelia amplexula Stirton $\equiv$ Xanthoparmelia amplexula (Stirton) Elix \& Johnston

Parmelia antleriformis Elix $\equiv$ Xanthoparmelia antleriformis (Elix) Elix \& Johnston

Parmelia australiensis Crombie $[\equiv$ Xanthoparmelia australiensis (Crombie) Hale]. Not present in Tasmania.

Parmelia bungendorensis Elix $\equiv$ Xanthoparmelia bungendorensis (Elix) Elix \& Johnston

Parmelia caperata (L.) Ach. Refers to Flavoparmelia haysomii (Dodge) Hale in Australia.

Parmelia concors Krempelh. $\equiv$ Parmotrema cristiferum (Taylor) Hale
Parmelia conferta Taylor $\equiv$ Hypogymnia billardierei (Mont.) R. Filson

Parmelia conlabrosa Hale. Not recorded for Tasmania by Elix \& Stevens (1979).

Parmelia conspersa (Ach.) Ach. Not present in Tasmania and generally misapplied in the past to many species of Xanthoparmelia. Two subspecific taxa, $P$. conspersa var. incisa (Taylor) Zahlbr. and P. conspersa var. laxa Mijll. Arg. are synonyms of Xanthoparmelia tasmanica (J.D. Hook. \& Taylor) Hale.

Parmelia cristifera Taylor $\equiv$ Parmotrema cristiferum (Taylor) Hale

Parmelia hookeri Taylor. The description in the original reference (Wilson 1893) suggests that this record refers to the common species Parmelina stevensiana Elix \& Johnston.

Parmelia hypoclystoides (Müll. Arg.) Gyelnik $\equiv$ Xanthoparmelia scabrosa (Taylor) Hale

Parmelia inaequalis Taylor $\equiv$ Pannoparmelia angustata (Pers. in Gaud.) Zahlbr.

Parmelia jelineckii Krempelh, EFlavoparmelia rutidota (J.D. Hook. \& Taylor) Hale

Parmelia laceratula Nyl. [ $\equiv$ Punctelia rudecta (Ach.) Krog.] Not present in Tasmania.

Parmelia moniliformis J.D. Hook. = Pannoparmelia angustata (Pers. in Gaud.) Zahlbr.

Parmelia mundata Nyl. var, pulverata Nyl. $\equiv$ Hypogymnia pulverata (Nyl.) Elix

Parmelia neorimalis Elix \& P. Armstrong $[=$ Xanthoparmelia neorimalis (Elix \& P. Armstrong) Elix \& Johnston]. Not present in Tasmania (Elix et al. 1986).

Parmelia ochroleuca Müll. Arg. $\equiv$ Flavoparmelia rutidota (J.D. Hook.\& Taylor) Hale

Parmelia olivacea (L.) Ach. Not present in Tasmania and erroneously applied to several brown species of Parmelia s. lat.

Parmelia omphalodes (L.) Ach. Refers to P. signifera Nyl, in Tasmania (Filson 1986).

Parmelia perforata (Wulfen) Ach. The description of the taxon referred to under this name by Wilson (1893) suggested it is Parmotrema reticulatum (Taylor) Choisy.

Parmelia perlata (Huds.) Ach. $\equiv$ Parmotrema chinense (Osbeck) Hale \& Ahti

Parmelia physodes var. sublugubris Müll. Arg. $\equiv$ Hypogymnia lugubris var. sublugubris (Müll. Arg.) Elix

Parmelia prolixa (Ach.) Malbr. $\cong$ Neofuscelia pulla (Ach.) Esslinger

Parmelia pruinata Müll. Arg. Refers to Parmelina stevensiana Elix \& Johnston in Tasmania (Elix \& Johnston 1986).

Parmelia quercina Willd. Not present in Tasmania. Record probably refers to Parmelina stevensiana Elix \& Johnston.

Parmelia reptans Kurok. [ $\equiv$ Xanthoparmelia reptans (Kurok, Elix \& Johnston]. Not present in Tasmania (Elix et al. 1986).

Parmelia rudecta Ach. Refers to Punctelia subflava (Taylor) Elix \& Johnston in Australia.

Parmelia saxatilis (L.) Ach. Not present in Tasmania (Galloway \& Elix 1983). 
Parmelia scabropustulata Elix $\equiv$ Xanthoparmelia scabrosa (Taylor) Hale

Parme lia splachnirima J.D. Hook. \& Taylor $\equiv$ Knightiella splachnirima (J.D. Hook. \& Taylor) Gyel nik

Parmelia stenophylla (Ach.) Heug. = Xanthoparmelia digitiformis (Elix \& P. Armstrong) R. Filson

Parmelia subalbicans Stirton. Not present in Tasmania.

Parmelia ubcaperatula Nyl. F Flavoparmelia rutidota

(J.D. Hook. \& Taylor) Hale

Parmelia subglabra Räsänen $\equiv$ Melanelia subglabra (Räsänen) Esslinger

Parmelia subprolixa Krempelh. $\equiv$ Neofuscelia pulla (Ach.) Esslinger

Parmelia tiliacea (Hoffm.) Ach. Not present in Tasmania. The name refers to Parmelina conlabrosa (Hale) Elix \& Johnston in Australia (Filson 1986).

Parmelia tinctina Mah. \& Gill. Refers to Xanthoparmelia isidiigera in Tasmania (Filson 1986).

Parmelia ulophylla (Ach.) F. Wilson $\equiv$ Punctelia subrudecta (Nyl.) Krog

Parmeliella amphibola (Knight) Müll. Arg. E Leioderma amphibolum (Knight) P.M. Jørg. \& D. Galloway

Parmeliella plumbea (Lightf.) Müll. Arg. Not present in

Tasmania. This name has probably been misapplied to species of Degelia and Coccocarpia.

Parmeliello triptophylla (Ach.) Müll. Arg. Jørgensen (1978) observed that no reliable Southern Hemisphere records of this European species have been traced.

Parmeliopsis semiviridis F. Muell. ex Nyl. [ Chondropsis semiviridis (F. Muell. ex Nyl.) Nyl. ex Crombie]. Not present in Tasmania.

Parmotrema cooperi (J. Stein \& Zahlbr.) Sérusiaux. An African species (Hale 1965, Krog \& Swinscow 1981) whose occurrence in Tasmania seems very doubtful.

Peltigera erumpens (Taylor) Vainio 프 Peltigera didactyla (With.) Laundon

Peltigera spuria (Ach.) DC. $\equiv P$. didactyla (With.) Laundon

Perforaria peponula (Müll. Arg.) Müll. Arg. $\equiv$ Coccotrema cucurbitula (Mont.) Müll. Arg.

Pertusaria ascripta Stirton. Not in Tasmania Previously misidentified for $P$. truncata Krempelh.

Pertusaria leioplaca (Ach.) DC. $\equiv P$. leucostoma (Bernh.) Mass. (Hanko 1983).

Pertusaria leioplaca var. octospora Nyl. $\equiv P$. alpina Hepp ex Ahles (Filson 1986).

Pertusaria nothofagi Zahlbr. $\equiv P$. truncata Krempelh.

Phaeographis dendritica (Ach.) Müll. Arg. Record doubtful.

Phlyctella wilsonii Müll. Arg. = Phlyctis subuncinata Stirton

Phyllopsora melanocarpa Müll. Arg. $\equiv$ Neophyllis melacarpa (F. Wilson) F. Wilson

Physcia astroidea (Clem.) Nyl. $\equiv P$. clementei (Sm.) Lynge

Physcia endococcinoides Poelt $\equiv$ Phacophyscia endococcinoides (Poelt) Esslinger

Physcia picta (Sw.) Nyl. I Dirinaria picta (Sw.) Clem. \& Shear

Physciopsis adglutinata (Flörke) Choisy $\equiv$ Hyperphyscia adglutinata (Flörke) Mayrhofer \& Poelt

Pilophorus conglomeratus F. Wilson $\equiv$ Metus conglomeratus (F. Wilson) D. Galloway \& P. James

Polychidium umhausense (Auersw.) Henssen. Misdetermined for Dendriscocaulon dendriothamnodes Dughi in D. Galloway.

Pseudocyphellaria amphisticta Knight. Record (by Bratt \& Cashin 1976) refers to $P$. argyracea (Delise) Vainio.

Pseudocyphellaria australiensis $\mathrm{H}$. Magn. $\equiv P$. neglecta (Müll. Arg.) H. Magn.

Pseudocyphellaria carpoloma (Delise) Vainio. Although recorded by several early workers (see Wetmore 1963), the species does not appear to occur in Tasmania and the name appears to have been misapplied to other taxa in the $P$. faveolata group.

Pseudocyphellaria cellulifera (J.D. Hook.) Gyeinik $\equiv$ $P$. faveolata (Delise) Malme.

Pseudocyphellaria cinnamomea (A. Rich.) Vainio. Recorded by early authors but all specimens examined to date are of $P$. dissimilis (Nyl.) D. Galloway \& P. James, a closely related, very common species.

Pseudocyphellaria delisea (Fée) D. Galloway \& P. James $\equiv$ P. glabra (J.D. Hook. \& Taylor) Dodge

Pseudocyphellaria dissimulata (Nyl.) Vainio. Refers to P. multifida (Nyl.) D. Galloway \& P. James

Pseudocyphellaria durvillei (Delise) Vainio. Misapplied to $P$. coronata (Müll. Arg.) Malme (see Galloway \& James 1977).

Pseudocyphellaria endochrysa (Delise) Vainio. Misapplied to $P$. coronata (Müll. Arg.) Malme.

Pseudocyphellaria flavicans (J.D. Hook. \& Taylor) Vainio. Not present in Tasmania and the name has probably been misapplied to $P$. colensoi (Church. Bab.) Vainio.

Pseudocyphellaria flotowiana (Laurer) Malme $\equiv$ $P$. billardierei (Delise) Räsänen

Pseudocyphellaria fossulata (Dufour) Malme $\equiv$ $P$. faveolata (Delise) Malme

Pseudocyphellaria freycinetii (Delise) Malme. A South Amercian species, widely confused with $P$. glabra (J.D. Hook. \& Taylor) Dodge in Australasia (Galloway 1985).

Pseudocyphellaria gilva (Ach.) Malme $\equiv \mathrm{P}$. crocata (L.) Vainio

Pseudocyphellaria hirta (Stirton) D. Galloway \& P. James $\equiv P$. coronata (Müll. Arg.) Malme

Pseudocyphellaria impressa (J.D. Hook, \& Taylor) Vainio $\equiv P$. faveolata (Delise) Malme

Pseudocyphellaria mougeotiana (Delise) Vainio $\equiv$ $P$. crocata (L.) Vainio

Pseudocizphellaria orygmaea (Ach.) Malme. Refers to $P$. coronata (Müll. Arg.) Malme

Pseudocyphellaria physciospora (J.D. Hook.) Malme $\equiv$ $P$. faveolata (Delise) Malme

Pseudocyphellaria polyschista (Meyen \& Flotow) Zahlbr. Listed for Tasmania by Filson (1986) but record appears doubtful.

Pseudocyphellaria prolificans (Nyl.) Vainio. Doubtful record presumed to be based on a misidentification.

Pseudocyphellaria richardi (Mont.) Massal. = $P$. billardierei (Delise) Räsänen 
Pseudocyphellaria subvariabilis (Nyl.) Vainio $\equiv P$. multifida (Nyl.) D. Galloway \& P. James

Pseudopyrenula glactina Shirley $\equiv$ Pyrenula galactina (Shirley) Kantvilas

Psoroma caliginosum Stirton. Refers to $P$. pholidotoides (Nyl.) Trevis. in Tasmania.

Psoroma contextum Stirton. Misapplied by Bratt \& Cashin (1976) to $P$. pholidotoides (Nyl.) Trevis.

Psoroma imbricatum (Nyl.) J. Murray $\equiv P$. asperellum Nyl.

Psoroma reticulatum (Hue) Zahlbr. $\equiv P$. pholidotoides (Nyl.) Trevis.

Psoroma sphinctrinum var. leprolomum Nyl. $\equiv P$. leprolomum (Nyl.) Räsänen

Psoroma sphinctrinum var. microphyllizans Nyl. $\equiv P$. microphyllozans (Ny1.) D. Galloway

Psoromaria descenderis (Nyl.) Nyl. = Psoromidium aleuroides (Stirton) D. Galloway

Ramalina brevis $\mathrm{F}$. Wilson var. brevissima $\mathrm{F}$. Wilson $\equiv$ R. fissa (Müll. Arg.) Vainio

Ramalina calicaris (L.) Fr. Refers to $R$. glaucescens Krempelh.

Ramalina ecklonii (Spreng.) Meyen \& Flotow $\equiv R$. celastri (Spreng.) Krog \& Swinsc.

Ramalina fastigiata (Pers.) Ach. Refers to $R$. glaucescens Krempelh.

Ramalina fraxinea (L.) Ach. var. brevissima $\mathrm{F}$. Wilson $\equiv$ R. fissa (Müll. Arg.) Vainio

Ramalina fraxinea f. platyna Nyl. $\equiv R$. fissa (Müll. Arg.) Vainio

Ramalina geniculata J.D. Hook. \& Taylor $\equiv R$. inflata (J.D. Hook. \& Taylor) J.D. Hook. \& Taylor

Ramalina inflata var. gracilis Müll. Arg. $\equiv R$. inflata (J.D. Hook. \& Taylor) J.D. Hook. \& Taylor

Ramalina leiodea (Nyl.) Nyl. $[\equiv R$. subfraxinea var. leiodea (Nyl.) Stevens]. Not present in Tasmania.

Ramalina membranacea (Laurer) Mont. Not present in Australia. Record probably refers to $R$. celastri (Sprengel) Krog \& Swinsc.

Ramalina pollinaria (Liljebl.) Ach. Misapplied to $R$. unilateralis $\mathrm{F}$. Wilson.

Ramalina pusilla le Prev. $\equiv R$. inflata ssp. australis N. Stevens

Ramalina tasmanica Nyl. $\equiv R$. inflata (J.D. Hook. \& Taylor) J.D. Hook. \& Taylor

Ramalina yemensis (Ach.) Nyl. $\equiv R$. celastri (Sprengel) Krog \& Swinsc.

Ramalina yemensis ssp. ovalis (J.D. Hook. \& Taylor) Zahlbr. $\equiv R$. celasiri ssp. ovalis (J.D. Hook. \& Taylor) N. Stevens

Siphula coriacea Taylor ex Nyl. Not present in Tasmania

Siphula dissoluta Nyl. Not present in Tasmania. Previously misapplied to some forms of $S$. decumbens Nyl. s. lat.

Siphula pteruloides Nyl. Misapplied to S. complanata. (J.D. Hook. \& Taylor) R Sant.

Siphula torulosa (Ach.) Nyl. Generally misapplied to S. decumbens Nyl. (see Mathey 1974).

Sphaerophorus australis Laurer $\equiv S$. melanocarpus (Sw.) DC.
Sphaerophorus compressus Ach. $\equiv S$. melanocarpus (Sw.) DC

Sphaerophorus fragilis (L.) Pers. Not present in Tasmania (see Kantvilas 1988).

Sphaerophorus globosus (Huds.) Vainio. Not present in Tasmania.

Stereocaulon colensoi Church. Bab. Not present in Tasmania.

Stereocaulon coralloides Fr. ( $\equiv S$. dactylophyllum Flörke). Not present in Tasmania.

Stereocaulon denudatum Flörke $\equiv S$. vesuvianum Pers.

Stereocaulon gracilescens Nyl. [झ Leprocaulon gracilescens (Nyl.) Lamb \& Ward]. Not present in Tasmania (Lamb \& Ward 1974).

Stereocaulon kilmandscharoense Stein $\equiv S$. vesuvianum Pers.

Stereocaulon leptaleum $\mathbf{N y l} . \equiv S$. corticatulum $\mathrm{Nyl}$.

Stereocaulon macrocarpum $\mathrm{Rich} . \equiv S$. ramulosum $(\mathrm{Sw}$.) Räusche1

Stereocaulon macrocarpoides Nyl. $\equiv S$. ramulosum (Sw.) Räuschel

Stereocaulon proximum Nyl. $\equiv S$. ramulosum (Sw.) Räuschel

Sticta cetrarioides J.D. Hook. $\equiv$ Heterodea muelleri (Hampe) Nyl.

Sticta cinereoglauca J.D. Hook. \& Taylor. Not present in Tasmania.

Sticta filicina Ach. [ES. filix (Sw.) Nyl.] Not present in Tasmania. Most Tasmanian records of species of Sticta with a green photobiont appear to be misidentifications of $S$. stipitata Knight.

Sticta filix (Sw.) Nyl. Not present in Tasmania (see above under S. filicina).

Sticta fragillima Church. Bab. Probably misapplied to Pseudocyphellaria dissimilis (Nyl.) D. Galloway \& P. James.

Sticta fulvocinerea Mont. Refers to Pseudocyphellaria glabra (J.D. Hook. \& Taylor) Dodge.

Sticta latifrons Rich. Not present in Tasmania.

Sticta linearis J.D. Hook. \& Taylor $\equiv$ Pseudocyphellaria billardierei (Delise) Räsänen

Sticta muelleri Hampe $\equiv$ Heterodea muelleri (Hampe) Nyl.

Sticta shirleyana Müll. Arg. $\equiv S$. stipitata Knight

Teloschistes chrysophthalmus var. sieberianus (Laurer) Müll. Arg. $\equiv T$. sieberianus (Laurer) Hillm.

Teloschistes flavicans var, subexilis (Nyl.) F. Wilson $\equiv$ T. spinosus (J.D. Hook. \& Taylor) J. Murray

Thelidea corrugata Hue $\equiv$ Knightiella splachnirima (J.D. Hook. \& Taylor) Gyelnik

Thysanothecium hyalinum Mont. \& Berk. $\equiv T$. scutellatum (Fr.) D. Galloway

Umbilicaria cinerascens (Ach.) Nyl. Misapplied to U. subglabra (Nyl.) Harm. (Blackman et al. 1974).

Umbilicaria proboscoidea (L.) Schrad. Not present in Tasmania (Blackman et al. 1974).

Usnea acromelana var. decipiens (Lamb) Lamb $\equiv U$. acromelana Stirton

Usnea acromelana var. inactiva Lamb $\equiv U$. torulosa (Müll. Arg.) Zahlbr. 
Usnea antactica Du Reitz. Not present in Tasmania (Waker 1985).

Usnea arida Motykd. Not present in Tasmania (G.N. Stevens, pers. comm.). Previous use of this name in the interatare refers mostly to $U$. leprosa Motyka.

Urrea aricilata Fr. Occurrence in Tasmania doubtful (C.N. Stevens, pers comm.).

Usnea harbara (L.) Wigg. Refers to U. scabrida Taylor

Usnea dasypoga (Ach.) Hornem. Not present in Tasmonia (G.N. Stevens, pers. comm.)

Uinet lasypogoides Nyl. ex Crombie. Hot present in Tasminia (G.N. Stevens, pers, comm.)

Usnea fexils Stirton. Occurrence in Tasmania doubtul. Usnea florida (L.) Wigg. Refers to $U$. scabrida Taylor. Usnea glomerata Motyka $\equiv U$. torulosa (Muil. Arg.) Zahisar.

Usnea longissima Ach. Uccurrence in lasmanta doubtful (G.N. Stevens, pers. comm.).

Usnea microcarpioides (Mül. Arg.) Motyka $\equiv$ U. ramulosissima Stevens \& Rogers

Usnea melaxamtha Ach. I $\equiv U$. aurantiaco-atra (Jacq.) Bory]. Not present in Tasmania (Walker 1985).

\section{ACKNOWLEDGEMENTS}

This checklist was made possible by the extensive help and support of many colleagues in Australia and abroad who identified or commented on my lichen collections, clarified nomenclatural probiems, provided copies of early literature, critically read drafts of the manuscript or otherwise provided encouragement in this project. I am particularly indebted to Dr T. Ahti, Dr A.W. Archer, Dr J.A. Elix, Dr R.B. Filson, Dr D.J. Galloway, Prof. Dr A. Henssen, Prof. Dr H. Hertel, Mr P.W. James, Dr S.J. Jarman, Ms J. Johnston, Prof. P.M. Jørgensen, Mr H.T. Lumbsch, Dr H. Mayrhofer, Dr R.W. Rogers, Dr H. Sipman, Dr G.N. Stevens, Dr L. Tibell, Mr D. Verdon and Dr A. Vezda.

\section{REFERENCES}

Ant, T, 1961: Taxonomic studies on reindeer lichens (Cladonia subgenus Cladina). Ann. Bot. Soc. Zool. Bot, Fenx. "Vanamo" 32: 1-160.

AHT, T', 1980: Taxonomic revision of Cladonia gracilis and its allies. Ann. Bot. Fennici 17: 195-243.

Arr, T, 1983: Taxonomic notes on some American species of the lichen genus Cladonia. Ann. Bot Fennici 20: $1-7$.

AirT, T, 1984: The status of Cladina as a genus segregated from Cladonia. Beih. Nova Hedwigia 79 $25-61$.

ARCHFR, A.W., 1980: A new Australian lichen: Cladonia kuringatensis. Muelleria 4: 273-275.
Usnea plicata (L.) Wigg, Occurrence in Tasmania doubtful (G.N. Stevens, pers. comm.).

Usneu rubescens Stirton $\equiv U$. rubicunda Stiton

Usnea sphacelata R.Br. Not present in Tasmania (Walker 1985).

Usnea straminea Müll. Arg. Not present in Tasmania (G.N. Stevens, pers. comm.).

Usnea subfusca Stirton. Refers to species of Usnea subgen. Neuropogon (G.N. Stevens, pers. comm.) of which only two laxa occur in Tasmanida, U. acromelana and $U$. subcapillaris.

Usnea tasmanica (Müll, Args) Zahbr. $\equiv U$. hailey (Stirton) Zahlbr.

Kanthoria ectanea (Ach.) Räsänen ex R. Filson $\equiv$ $X$ ligulata (Körber) P. James in Australia.

Xanthoria lychnea (Ach.) Fr. I $X$. candelaria (L.) Th. Fr.]. Not present in Tasmania.

Xanthoria spinosa (J.D. Hook. \& Taylor) Du Rietz $\equiv$ Teloschistes spinosus (J.D. Hook. \& Taylor) J. Murray. Kanthoria spinulosa (Krempelh.) Hillm. $=$ Teloschistes spinosus (J.D. Hook. \& Taylor) J. Murray.

ArCher, A.W., 1982: A new Australian lichen: Cladonid sulcata. Muelleria 5: 115-117.

Archer, A.W., 1984: Three new Australian lichens: Cladonia celata, C. praetermissa and C. wilsonii. Muelleria 5: 271-275.

Archer, A.W., 1985: Two new lichens: Cladonia bimberiensis and $C$. weymouthii. Muelleria 6: 93-95.

Archer, A.W., 1986: The chemistry and distribution of Cladonia capitellata (J.D. Hook. \& Taylor) Church. Bab. (Lichens) in Australia. Proc. Linn. Soc. N.S.W 108: $191-194$.

Archer, A.W., 1987: Two new lichens: Cladonia squamosula var. subsquamosula and $C$. sulcata var. striata with notes on chemotaxonomy within the species. Muelleria 6: 383-388.

ARCHER, A.W., 1988a: The lichen genus Cladonia section Cocciferae in Australia. Proc. Linn. Soc: N.S.W. 110:205-213.

Archer, A.W., 1988b: Two new lichens: Cladonia paeminosa and $C$. humilis var. bourgeanica. Muelleria 7: $1-5$.

Archer, A.W. \& Bartlett, J.K., 1986: New species and distributions of the lichen genus Cladonia in New Zealand together with a revised key. N.Z. J. Bot. 24: $581-587$.

Arvidsson, L., 1982: A monograph of the lichen genus Coccocarpia. Opera Bot. 67: $1-96$.

Arvidsson, L. \& Galloway, D.J., 1979: The lichen genus Coccocarpia in New Zealand. Bot. Notiser 132: $239-246$

Arvinsson, L. \& Galloway, D.J., 1981: Degelia, a new lichen genus in the Pannariaceae. Lichenologist 13: $27-50$.

Babington, C. \& Mitten, W., 1859: Lichenes. In Hooker, J.D. (Ed): FLORA TASMANIAE, vol.T1. Lovell-Reeve, London: 343-354. 
Blackman, A.J., Bratt, G.C. \& Cashin, J.A., 1973: Distribution and chemistry of Heteroded muelleri. Bryologist 76: 410-413.

Blackman, A.J., Bratr, G.C. \& Cashin, J.A., 1974: Umbilicariaceae in Tasmania. Lichenologist 6 : 112-114.

Bratt, G.C., Blackman, A.J. \& Cashin, J.A., 1976: The genus Anzia in Tasmania. Lichenologist 8: 69-77

Bratt, G.C. \& Cashin, J.A., 1975: Additions to the lichen flora of Tasmania I. Pap. Proc. R. Soc. Tasm. 109: 17-20.

Bratt, G.C. \& Cashin, J.A., 1976: Additions to the lichen flora of Tasmania II. Pap. Proc, $R$, Soc. Tasm. 110: 139-148.

Christensen, S.N., 1987: Morphological and chemical variation in the Cladonia macilentalbacillaris aggregate in Denmark. Lichenologist 19: 61-69.

Crombie, J.M., 1880: Enumeration of lichens in the herbarium of Robert Brown, with descriptions of new species. J. Linn. Soc. Lond. (Botany) 17: 390-401.

Degelius, G., 1974: The lichen genus Collema with special reference to extra-European species. Symb. Bot. Upsal. 20: $1-215$.

Döbbeler, P., 1981: Moosbewohnende Ascomyceten V. Die auf Dawsonia vorkommenden arten der Botanischen Staatssammlung München. Mitt. Bot. Müchen 17: 393-474.

Döbbeler, P., Poelt, J. \& Vezda, A., 1985: Lopadium hepaticola spec. nov. ein moosparasitisches echtes Lopadium von der Sudhalbkugel. Herzogia 7: 8l-91.

ElIX, J.A., 1979: A taxonomic revision of the lichen genus Hypogymnia in Australasia. Brunonia 2: $175-245$.

Elix, J.A., 1981a: Further new species of Parmelia (Lichens) from Australia. Aust. J. Bot. 29: 17-23.

ELIX, J.A., 1981b: New species of Parmelia subgen. Xanthoparmelia (Lichens) from Australia and New Zealand. Aust. J. Bot. 29: 349-376.

Elix, J.A. \& Armstrong, P.M., 1983: Further new species of Parmelia subgen. Xanthoparmelia (Lichens) from Australia and New Zealand. Aust. J. Bot. 31: $467-483$.

Elix, J.A. \& Johnston, J., 1986: New species of Parmelina (Lichenised Ascomycotina) from Australia and New Zealand. Brunonia 9: 155-161.

Elix, J.A. \& Johnston, J., 1987: New species and new records of Xanthoparmelia (lichenized Ascomycotina) from Australia. Mycotaxon 29: 359-372.

Elix, J.A. \& Johnston, J., 1988: New species in the lichen family Parmeliaceae (Ascomycotina) from the southern hemisphere. Mycotaxon 31: 491-510.

Elix, J.A., Johnston, J, \& Armstrong, P.M., 1986: A revision of the lichen genus Xanthoparmelia in Australasia. Bull. Br. Mus. Nat. Hist. (Bot.) 15: 163-362.

Elix, J.A. \& Stevens, G.N., 1979: New species of Parmelia (Lichens) from Australia. Aust. J. Bot. 27: 873-883.

Elix, J.A. \& Streimann, H., 1982: New lichen records for Australia. J. Hattori Bot. Lab. 51: 69-97.

Esslinger, T.L., 1977: A chemosystematic revision of the brown Pameliae. J. Hathot Bot. Lab. 42: $1-211$.

FILSON, R.B., 1969: A review of the genera Teloschistes and Xanthoria in the lichen family Teloschistaceae in Austratia. Muelleria 2: 65 115.

Filson, R.B., 1978: A revision of the genus Heterodea. Lichenologist 10: 13-25.

Fllson, R.B., 1981: A revision of the lichen genus Cladia. J. Hattori Bot. Lab. 49: 1-75.

Frlson, R.B., 1982: A contribution on the genus Parmelia (Lichens) ir southern Australia. Aust. J. Bot. 30: $511-582$.

FILSON, R.B., 1983: CHECKLIST OF AUSTRALIAN LICHENS. National Herbarium of Victoria, Melbourne.

FILson, R.B., 1984: Xanthoparmelia elixit (Lichens), a new species from Australia and New Zeaiand. Brunonia 7: 203-205.

FILSON, R.B., 1986: CHECKLIST OF AUSTRALIAN LICHENS. Second edition, National Herbarium of Victoria, Melbourne.

FILSON, R.B., 1988a: The lichen genera Heppia and Peltula in Australia. Muelleria 6: 495-517.

FILSON, R.B., 1988b: CHECKLIST OF AUSTRALIAN LICHENS. Third edition, National Herbarium of Victoria, Melbourne.

Filson, R.B. \& ROGERS, R.W, 1979: LICHENS OF SOUTH AUSTRALIA. South Australian Government Printer, Adelaide.

Galloway, D.J., 1974: A bibliography of New Zealand lichenology. N.Z. J. Bot. 12: 397-422.

Galloway, D.J., 1978: Anzia and Pannoparmelia (Lichenes) in New Zealand. N.Z. J. Bot. 16: 261-270.

Galloway, D.J., 1980a: Notes on the lichen genus Baeomyces in New Zealand. Bot. Notiser 133: 77-83.

Galloway, D.J., 1980b: The lichen genera Argopsis and Stereocaulon in New Zealand. Bot. Notiser 133; 261-279.

Galloway, D.J., 1980c: Xanthoparmelia and Chondropsis (Lichenes) in New Zealand. N.Z. J. Bot. 18: 525-552.

Galloway, D.J., 1981: The lichen genus Lobaria in New Zealand. Nova Hedwigia 34: 317-331.

Galloway, D.J., 1983a: Nomenclatural notes on Pseudocyphellaria I. The identity of Pseudocyphellaria neglecta. Lichenologist 15: 307-308.

Galloway, D.J., 1983b: New taxa in the New Zealand lichen flora. N.Z. J. Bot. 21: 191-200.

Galloway, D.J., 1985a: Nomenclature notes on Pseudocyphellaria II. Some southern hemisphere taxa. Lichenologist 17: 303-307.

Galloway, D.J., 1985b: FLORA OF NEW ZEALAND: LICHENS. Government Printer, Wellington.

Galloway, D.J., 1988: Studies in Pseudocyphellaria (lichens) I. The New Zealand species. Bull. Br. Mus. Nat. Hist. (Bot.) 17: 1-267.

Galloway, D.J. \& Bartlett, J.K., 1983: The lichen genus Thysanothecium Mont. \& Berk. in New Zealand. Nova Hedwigia 36: 381-398.

Galloway, D.J. \& Bartlett, J.K., 1986: Arthroraphis 
Th. Fr. (lichenised Ascomycotina) in New Zealand. N.Z. J. Bot. 24: 393-402.

Gallow Ay, D.J. \& Elix, J.A., 1980: Knightiella Müll. Arg., a monotypic lichen genus from Australasia. N.Z. J. Bot - 18; $48 \mathrm{I}-486$.

Gallow Ay, D.J. \& Elix, J.A., 1983: The lichen genera Parme lia Ach. and Punctelia Krog in Australasia. N.Z. J. Bot. 21: $397-420$.

Gallow Ay, D.J. \& Elix, J.A., 1984: Additional notes on Parmelia and Punctelia (lichenised Ascomycotina) in Australasia. N.Z. J. Bot. 22: 441-445.

GAllowAY, D.J. \& JAMES, P.W., 1977: Pseudocyphellaria berberina: notes on its discovery and synonymy. Lichenologist 9: 95-105.

Gallow Ay, D.J. \& James, P.W., 1980: Nomenclatural notes on Pseudocyphellaria in New Zealand. Lichenologist 12: 291-303.

Galloway, D.J. \& James, P.W., 1985: The lichen genus Psoromidium Stirton. Lichenologist 17: 173-188.

Gallow AY, D.J. \& James, P.W., 1987: Metus, a new austral lichen genus and notes on an australasian species of Pycnothelia. Notes R.B.G. Edinb. 44: $561-579$.

Galloway, D.J., James, P.W. \& Wilkins, A.L., 1983 Further nomenclatural and chemical notes on Pseudocyphellaria in New Zealand. Lichenologist 15: 135-146.

Galloway, D.J. \& Jørgensen, P.M., 1987: Studies in the lichen family Pannariaceae II. The genus Leioderma Nyl. Lichenologist 19: 345-400.

Galloway, D.J., Lamb, I.M. \& Bratt, G.C., 1976: Two new species of Stereocaulon from New Zealand and Tasmania. Lichenologist 8: 61-67.

Hafellner, J., 1981: Monographie der Flechtengattung Letrouitia (Lecanorales, Teloschistineae). Nova Hedwigia 35: 645-729

HALE, M.E., 1965: A monograph of Parmelia subgenus Amphigymnia. Contrib. U.S. Nat. Herb. 36: 193-358.

HALE, M.E., 1975: A monograph of the lichen genus Relicina (Parmeliaceae). Smithson. Contrib. Bot. 26 $1-32$.

HaLe, M.E., 1976: A monograph of the lichen genus Pseudoparmelia Lynge. Smithson. Contrib. Bot. 31 $1-62$.

HALE, M.E., 1988: A monograph of the lichen genus Parmelia Acharius sensu stricto (Ascomycotina: Parmeliaceae). Smithson. Contrib. Bot, 66: 1-55.

Hampe, E., 1852: Plantae Muellerianae, Beitrag Flora Sudaustraliens. Lichenes, Linnaea 25: 709-712.

HANKO, B., 1983: Die chemotypen der flechtengattung Pertusaria in Europa. Bibliotheca Lichenologica 19: 1-297.

Hawksworth, D.L., 1972: Regional studies in Alectoria (Lichenes) 1I. The British species. Lichenologist 5: $181-261$.

HAYWARD, G.C., 1977: Taxonomy of the lichen families Graphidaceae and Opegraphaceae in New Zealand. N.Z. J. Bot. 15: 565-584.

Henssen, A, 1963: Eine revision der flechtenfamilien Lichinaceae und Ephebaceae. Symb. Bot. Upsal. 18 : $1-123$.
Henssen, A., 1969: Three non-marine species of the genus Lichina. Lichenologist 4: 88-98.

Henssen, A. \& Kantvilas, G., 1985: Wawea fruticulosa, a new genus and species from the Southern Hemisphere. Lichenologist 17: 85-97.

Henssen, A., Vobis, G. \& Renner, B., 1982: New species of Roccellinastrum with an emendation of the genus. Nord. J. Bot, 2; 587-599.

Hertel, H., 1984: Über saxicole, lecideoide Flechten der Subantarktis. Beih. Nova Hedwigia 79: $399-499$.

Hertel, H., 1987: Bemerkenswerte Funde südhemisphärischer, saxicoler Arten der Sammelgattung Lecidea. Mitt. Bot. München. 23: 321-340.

James, P.W. \& Henssen, A., 1975: A new species of Psoroma with sorediate cephalodia. Lichenologist 7 : 143-147.

Jørgensen, P.M., 1978: The lichen family Pannariaceae in Europe. Opera Bot. 45: 1-124.

Kantvilas, G, 1985: Studies on Tasmanian rainforest lichens. Unpubl. Ph.D. thesis, Univ. Tasm.

Kantvilas, G., 1987: Siphula jamesii, a new lichen from south-western Tasmania. Nord. J. Bot. 7: 585-588.

Kantvilas, G, 1988: A re-examination of John Shirley's collection of Tasmanian lichens. Pap. Proc. R. Soc Tasm. 122: 59-67.

Kantvilas, G. \& Elix, J.A., 1987: A new species of Cladia (lichenized Ascomycotina) from Tasmania. Mycotaxon 29: 199-205

Kantvilas, G. \& James, P.W., 1987: The macrolichens of Tasmanian rainforest: key and notes. Lichenologist 19: $1-28$.

Kantvilas, G., James, P.W. \& Jarman, S.J., 1985 Macrolichens in Tasmanian rainforests. Lichenologist 17: $67-83$

Kantrilas, G. \& JARMAN, S.J., 1988: Lichens of buttongrass (Gymnoschoenus) moorland in Tasmania. Pap. Proc. R. Soc. Tasm. 122: 1-17.

Kantvilas, G, \& Vezda, A., 1988; A new lichenised species of Arthonia from south-westem Tasmania. Aust. Syst. Bot. 1: 189-190.

KärnEFELT, I., 1979: The brown fruticose species of Cetraria. Opera Bot. 46: 1-150.

KroG, H. \& Swinscow, T.D.V., 1981: Parmelia subgenus Amphigymnia (lichens) in East Africa. Bull. Br. Mus. Nat. Hist. (Bot.) 9: 143-231.

LAMB, I.M. \& WARD, A., 1974: A preliminary conspectus of the species attributed to the imperfect lichen genus Leprocaulon Nyl. J. Hattori Bot. Lab. 38: 499-553.

LAUNDON, J.R., 1981: The species of Chrysothrix. Lichenologist 13: 101-122.

Lumbsch, H.T., 1987: Eine neue Subspecies in der Flechtengattung Diploschistes aus der Südhemisphare. Herzogia 7: 601-608.

LumBsCH, H.T., 1988a: Additional lichen records from Australia. 2. Austral. Lichen. Newsletter 22: 6-7.

LumBsch, H.T., 1988b: Additional lichen records from Australia. 3. Austral. Lichen. Newsletter 23: 3-4.

Martin, W., 1962: Notes on some New Zealand species of Cladonia with descriptions of two new species and one new form. Trans. Roy. Soc. N.Z. (Bot.) 2: $39-44$ 
Mathey, A., 1974: Contribution à l'étude du genre Siphula (Lichens) en Afrique. Nova Hedwigia 22: $795-878$.

MAYRHOFER, H., 1983: The saxicolous species of Rinodina in New Zealand. Lichenologist 15: 267-282.

Mayrhofer, H., 1984: The saxicolous species of Dimelaena, Rinodina and Rinodinella in Australia. Beih. Nova Hedwigia 79: 511-535.

Müller, J., 1887: Revisio lichenum australiensium Krempelhuberi. Flora 70: 113-118.

Ohlsson, K., 1974: A revision of the lichen genus Sphaerophorus. Unpubl. thesis, Michigan State Univ.

Petersen, R.H. \& Kantvilas, G., 1986: Three lichenforming Clavarioid fungi from Tasmania. Aust. J. Bot. 34: $217-222$.

RäsÄnen, V., 1944: Lichens novi II. Soumal eläin-ja Kasvit, seur. van. Julk. 21: 1-6.

Rogers, R.W., 1982: The corticolous species of Haematomma in Australia. Lichenologist 14: 115-129.

Rogers, R.W., 1985: Additional notes on Haematomma in Australia. Lichenologist 17: 207-309.

Rogers, R.W., 1986: The genus Pyxine (Physciaceae, Lichenized Ascomycetes) in Australia. Aust. J. Bot. 34: $131-154$.

Shirley, J., 1893: A list of the known lichens of Tasmania. Pap. Proc. R. Soc. Tasm. (1892): 179-191.

Shirley, J., 1894: Notes on Tasmanian lichens. Pap. Proc. R. Soc. Tasm. (1893): 214-219.

SiPMAN, H.J.M., 1983: A monograph of the lichen family Megalosporaceae. Biblioth. Lich. 18: 1-241.

SiPMAN, H.J.M., 1986: Additional notes on the lichen family Megalosporaceae. Willdenowia 15: 557-564.

STEnroos, S., 1988: The family Cladoniaceae in Melanesia 3. Cladonia sections Helopdium, Perviae and Cladonia. Ann. Bot. Fennici 25: 117-148.

Stevens, G.N., 1986: Three new saxicolous Ramalinae from Australia. Lichenologist 18: 183-189.

Srevens, G.N., 1987: The lichen genus Ramalina in Australia. Bull. Br. Mus. Nat. Hist. (Bot.) 16: 107-223.

Tehler, A., 1983: The genera Dirina and Roccellina (Roccellaceae). Opera Bot. 70: 1-86.

Trezelt, L., 1987: Australasian Caliciales. Symb. Bot. Upsal, 27: 1-279.

TIMDAL, E., 1984: The genus Hypocenomyce (Lecanorales, Lecideaceae) with special emphasis on the Norwegian and Swedish species. Nord. I. Bot. 4: $83 \cdots 108$.

VEZDA, A., 1977: Flechtensystematische studien X. Conotremopsis weberiana gen. novum et sp. nova, eine neue Flechte aus der Familie Ostropaceae. Folia Geobot. Phytotax., Praha 12: 313-316.

VEZDA, A. \& KAnTvilAs, G., 1988: Sarrameana tasmanica, a new Tasmanian lichen. Lichenologist 20: 179-182.

WALKER, F.J., 1985: The lichen genus Usnea subgenus Neuropogon. Bull. Br. Mus. Nat. Hist. (Bot.) 13: $1-130$.

Wetmore, C.M., 1963: Catalogue of the lichens of Tasmania. Rev. Bryol. et Lichenol. 32: 223-264.

White, F.J. \& J JMes, P.W., 1988: Studies on the genus Nephroma II. The southern temperate species. Lichenologist 20: 103-166.

Wilkins, A.L. \& James, P.W., 1979: The chemistry of Pseudocyphellaria impressa s. lat. in New Zealand. Lichenologist 11: 271-281.

Wilson, F.R.M., 1893: Tasmanian lichens. Pap. Proc. $R$. Soc. Tasm. (1892): 133-178.

Yoshimura, I., 1973: Notes on Gymnoderma melacarpum comb. nov. J. Jap. Bot. 48: 283-288.

(accepted 18 August 1989) 